\title{
A conspectus of the flower fly genus Allograpta (Diptera: Syrphidae) with description of a new subgenus and species
}

\author{
XIMO MENGUAL ${ }^{1}$, CARLOS RUIZ $^{2}$, SANTOS ROJO $^{1}$, GUNILLA STÅHLS $^{3} \&$ \\ F. CHRISTIAN THOMPSON ${ }^{4}$ \\ ${ }^{1}$ Departamento de Ciencias Ambientales y Recursos Naturales, Instituto Universitario CIBIO, Universidad de Alicante, Apdo 99. \\ E-03080, Alicante, Spain.E-mail: xmengual@ua.es, santos.rojo@ua.es \\ ${ }_{2}^{2}$ Grupo de Investigaciones Entomológicas, Sección de Entomología, Departamento de Biología, Universidad del Valle, Calle 13 No. \\ 100-00, A. A. 25623, Ciudad Universitaria Meléndez, Colombia.E-mail: carlosruiz78@gmail.com \\ ${ }^{3}$ Entomology Department, Finnish Museum of Natural History, PO Box 17, , FI-00014 University of Helsinki, Finland. \\ E-mail: gunilla.stahls@helsinki.fi \\ ${ }^{4}$ Department of Entomology, NHB-0169, Smithsonian Institution, Washington, D. C. 20560, USA [formerly Systematic Entomology \\ Laboratory, PSI, Agricultural Research Service, U. S. Department of Agriculture] . E-mail: thompsonf@si.edu
}

\begin{abstract}
A new subgenus [Allograpta (Costarica Mengual \& Thompson), type Allograpta zumbadoi Thompson], and one new species [Allograpta (Costarica) nishida Mengual \& Thompson; type-locality: Costa Rica, type-depository: Instituto Nacional de Biodiversidad de Costa Rica] of flower flies (Diptera: Syrphidae) are described from the Neotropical biotic region. A checklist of the world species of Allograpta including synonyms is provided, and a key to and diagnoses of the subgenera are also supplied. The phylogenetic relationships among Allograpta species, representing all hitherto detected morphological diversity of the genus, and related genera were studied under parsimony based on morphological characters.
\end{abstract}

Key words: Costarica, identification key, Syrphinae, taxonomy

\section{Introduction}

Allograpta flower flies are common in all areas except the extreme north and south and most of the Palaearctic Region. These flies are pollinators and show considerable variation in morphological characters, such as color pattern and head shape. The immatures of most species are predators of hemipteran pests (see Rojo et al. 2003 for review of published prey records). Recent studies have revealed that some species are secondarily leaf-miners (Nishida et al. 2002), stem-borers (Zuijen \& Nishida 2009) or pollen-feeders (Weng $\&$ Rotheray 2009). This major and important shift in biology from predation to phytophagy has highlighted the need for a reassessment of the genus Allograpta. This is the first review of the current classification of the group including a new name for the group of stem-boring species. All subgenera and species groups are morphologically diagnosed, and all names of included species in each subgenus or comparable grouping are given, as well as a key to subgeneric taxa. In order to provide a basis for further studies, morphological characters of Allograpta species and related genera, representing all known morphological diversity, were studied to explore their phylogenetic information content.

Other reports on the genus are in process. Mengual et al. (2008b) examined DNA sequence data and the immature stages will be analyzed. Then a phylogenetic assessment based on the combined dataset may provide resolution upon which to base a new and improved classification. 


\section{History of the classification of Allograpta and related groups}

Allograpta was established for the Nearctic species, Scaeva obliqua Say, as Osten Sacken felt that this species "... cannot well be placed in any of the existing genera of this group" (Osten Sacken 1875: 63). He noted differences from Sphaerophoria (small genitalia), Toxomerus [as Mesograpta] (male vertical triangle and mesonotal coloration) and while he said that "... it might be placed among the species of Syrphus with a linear abdomen," he noted that "Scaeva obliqua possesses in the structure of the eyes of the male, and in the peculiar markings of its abdomen, sufficient characters of its own." His name, Allograpta, "... is given in allusion to the peculiar coloration of the typical species." He provisionally placed Scaeva emarginata Say [now Epistrophella Dušek \& Laská (1967) or Epistrophe (Epistrophella) Vockeroth (1969, 1992)] in Allograpta and suggested "... more than one Syrphus from Mexico and the West Indies belongs to the same group; as for instance S. delineatus Macq., but, of course, it is impossible to judge from descriptions alone." Later Osten Sacken (1877: 331) described a second species from California [Allograpta fracta Osten Sacken]. The peculiar characters that Osten Sacken mentioned for this genus, the male eye structure (dorsal ommatidia about twice as large as ventral ones) and the combination of oblique lateral and straight medial vittae on 4th and 5th terga are no longer considered significant as these characters vary considerably among species within the currently defined concept of Allograpta.

Williston was the first specialist on New World flower flies, with his $\mathrm{PhD}$ thesis being a revision of the North American species (Williston 1887). His concept of Allograpta (Williston 1882: 310, 1887: 96, 1888: 50, 1896: 87, 1908: 255) was the same as Osten Sacken's. However, in his last key (1908: 255) he noted that the group was "feebly characterized." Bigot (1883: 256) likewise considered the group as dubiously distinct.

Lynch Arribalzaga (1892: 58) recognized the genus and included the first Neotropical representative (hortensis Philippi).

While Shannon $(1922 ; 1923)$ made major improvements in the classification of New World flower flies, his concept of Allograpta remained the same as Osten Sacken's and Williston's. He used the character of a premarginal sulcus on the abdomen (emarginate abdomen) in his first couplet to the key to genera of Syrphinae to separate the tribe Melanostomini from the tribe Syrphini. However he noted that Allograpta and about half the species of Syrphus also lacked the premarginal sulcus on the abdomen. Later when Shannon (1927: 2) described Claraplumula and Fazia, he separated these two genera along with Scaeva and Syrphus from Allograpta by the well-developed calypter and plumula.

Curran (1925b: 19, couplet 17) seems to be the first to have used the abdominal premarginal sulcus character to separate various Syrphini genera. Allograpta was grouped with those without a premarginal sulcus on the abdomen, but otherwise his definition of Allograpta remained the same as previous definitions (also Curran 1934: 255). Nevertheless, Curran greatly expanded the number of species included in Allograpta from 3 to 13, providing a key to them in 1932.

Fluke, in a series of papers $(1931,1933,1935)$, addressed the classification of a number of syrphine genera, but did not mention Allograpta. In his 1935 paper, he introduced the character of the pilosity of the metasternum, whether bare or pilose. In 1942, Fluke revised the Neotropical Syrphini, dealing with Allograpta and its related groups for the first time. He separated Allograpta (current definition) from other genera by its pilose metasternum and lack of a premarginal sulcus on the abdomen. He recognized Allograpta, Claraplumula and Epistrophe, with subgenus Fazia, although "Epistrophe, Fazia and Allograpta are scarcely separable." This classification was reasonable except that Fluke included some species of Ocyptamus in his Epistrophe. He also noted that "A careful study of the genitalia of the males of Allograpta, Fazia, Claraplumula and Epistrophe shows the close relationship of these groups." Later he published his work on the male genitalia (Fluke 1950), where he restricted the taxonomic concept of Epistrophe, recognized Allograpta, Claraplumula, and Fazia, but left a few species of Allograpta in Stenosyrphus (Episyrphus and Metepistrophe).

In 1938, Enderlein published contributions to the knowledge of Syrphidae, which unfortunately was more of obfuscation than enlightenment. For the Allograpta group, he recognized Fazia, which he placed in the 
Syrphini. Allograpta and his new genus Chasmia were placed in the Toxomerini. His Chasmia is a synonym of Allograpta, but he included one species (quadricincta) of Toxomerus in it. In Allograpta, he included a species of Dasysyrphus (munzoi = Dasyrphus pauxillus) and two species of Ocyptamus (delimbata and schoenemanni). His Fazia included only species of Allograpta, sensu stricto.

Frey (1946) in his revision of the syrphine genera followed previous workers in using the presence or absence of the premarginal sulcus of the abdomen to separate the genera related to Epistrophe. However, he separated Epistrophe and other genera from the Allograpta related groups by the lack of bright sharply defined yellow maculae on the pleuron. Then he divided the genera related to Allograpta first on the basis of facial shape as expressed as the shape of the oral opening (short and oval versus long and narrow). Finally Sphaerophoria was separated on its traditional characters of enlarged male genitalia. What is clear is that Frey apparently did not know the type of Allograpta as he would have realized that its facial shape is the same as his new genus, Miogramma. Under Allograpta, Frey merely wrote that the abdominal pattern well characterizes the genus. Under his Miogramma, he included a long discussion of how the group differs from Epistrophe, Sphaerophoria, Xanthogramma, Obliosyrphus, Ischiodon, Meligramma et alia, but no mention of Allograpta. Likewise, Microsphaerophoria was contrasted with Sphaerophoria, not Allograpta.

Hull (1949a), in his review of New World Baccha, included a key to the genera and subgenera related to Baccha. He separated Allograpta and Epistrophe from Baccha on the basis of the pilose metasternum and separated Rhinoprosopa from Baccha on the basis of its facial shape and widely separated antennal pits. However, his major work on the classification of flower flies (1949b) treated Allograpta and related groups as subgenera of Epistrophe. Unfortunately his concept of Epistrophe is "ein mischmash" [as Meigen (1822: 382) wrote of Fabricius' Eristalis]. Hull divided the syrphine genera first on whether there was a premarginal sulcus on the abdomen and then whether the metasternum was bare or pilose (Hull 1949b: 281, couplets 2-4). Then, after excluding Afrosyrphus and Paragus on antennal length, he was left with his Epistrophe and Sphaerophoria (1949b: 282. couplets 25-29). Sphaerophoria was separated by its traditional characters, especially enlarged male genitalia. Under Epistrophe he recognized as subgenera: 1) Epistrophe [= Epistrophe sensu stricto]; 2) Allograpta [= Allograpta sensu stricto], 3) Fazia [= Fazia, in part], 4) Metallograpta [= Fazia, in part], 5) Metepistrophe [= Fazia, in part], 6) Chasmia [= Fazia, in part], 7) Claraplumula [= Claraplumula], 8) Allograptina [= Argentinomyia], 9) Phalacrodira [= Parasyrphus $]$ and he did not recognize the available names Euryepistrophe, Heterepistrophe and Episyrphus. He separated these subgenera mainly on facial shape, abdominal pattern and shape. Allograpta was separated from Epistrophe on abdominal pattern as he explained "I cannot see any valid distinctions in the genotype except upon the abdominal pattern for this group." Then he named Metallograpta for those Allograpta species with a projecting face; and Metepistrophe for those "species of Epistrophe in which the epistoma juts forward." He separated this new subgenus from Fazia based on abdominal shape (oval in Fazia, elongate in Metepistrophe).

Bankowska (1962), when revising the Palaearctic species of Sphaerophoria, re-examined the relationships of Syrphus javanus Wiedemann, a species placed in Sphaerophoria by some earlier authors. She concluded that javanus could not be placed in Sphaerophoria, Xanthogramma or Ischiodon. So, being unaware of Allograpta and the work of Frey (1946), she erected a new genus for javanus, Helenomyia, which became a junior objective synonym of Miogramma Frey and a subjective synonym of Allograpta. Apparently Shiraki (1963) also recognized the distinctiveness of javanus as he placed a synonym (nakamurae Matsumura) of it in Paraxanthogramma, but never formally published a description of the genus. The name was subsequently used in a work on the biological control of aphids in Taiwan by Tao \& Chiu (1971).

Vockeroth $(1969,1973)$ was responsible for the current concept of Allograpta. His 1969 paper included 55 Allograpta species representing almost all biogeographic regions and he was the first to recognize that Old World species belonged to the New World genus. Additionally, he discussed the zoogeography of the taxon. He based his concept of Allograpta on the male genitalia ["Despite the great variation in many characters the male terminalia are very distinctive and separate the genus sharply from all others"]. While Vockeroth did not explicitly define Allograpta in terms of male genitalic characters, the key characters were 1) the superior lobes 
fused to the hypandrium (in most syrphines these are articulating) and 2) basal part of the aedeagus enlarged and with dense black denticles (figs. 11-16).

\section{Identification}

The Nearctic species were treated by Vockeroth (1992). The last key to the Neotropical species of Allograpta is by Fluke (1942), but is inadequate as he mixed the species of Allograpta and Ocyptamus among his "Epistrophe" and did not include Rhinoproposa. Rhinoprosopa was treated as part of the genus Baccha by Hull (1949a: 101, couplets 3-5). Frey (1946: 166) provided a key to the Old World species of Allograpta as his Miogramma.

\section{Format}

Terminology follows Thompson (1999b). The classification followed here is that of the BioSystematic Database of World Diptera (Evenhuis et al. 2008) and follows from that of Vockeroth (1969) and the various regional Diptera catalogs (Knutson et al. 1975; Peck 1988; Smith \& Vockeroth 1981; Thompson et al. 1976; Thompson \& Vockeroth 1989. Also see older catalogs such as Harris 1835; Osten Sacken 1878; Aldrich 1905; Kertész 1910; Fluke 1956-7). We recognize that some alternatives to this classification scheme can be considered following recently published results of Mengual et al. (2008a, b), but these are based only on molecular characters and other proposals are forthcoming. So, for uniformity and universality, we here accept the traditional classification of flower flies. The format for and abbreviations found in the generic synonymies follow the BioSystematic Database of World Diptera (BDWD, Thompson 1999a). The acronyms used for collections likewise follow BDWD standards and their equivalents are given in the acknowledgments. In the species lists, the use of an asterisk $(*)$ means that material of the species was examined and two asterisks $(* *)$ means type material of the name was examined. The species are listed alphabetically by biotic regions. For each species we provide the valid epithet, the author, year and page number of the original description followed by the original genus in parenthesis and the distribution. If synonyms exist, they are given in brackets after the distribution.

\section{Phylogeny}

Recent improvements in laboratory techniques have made it feasible to employ an increasingly higher number of genes for the study the phylogenetic relationships of various insect groups (Bybee et al. 2004; Brammer \& von Dohlen 2007; Kutty et al. 2007; Schuh et al. 2009). The first phylogeny of the predatory flower flies (subfamily Syrphinae) based on molecular characters was published (Mengual et al. 2008a). It was the first time that the genus Allograpta was included in a molecular study. The results of the study brought light on the relationships among Syrphinae genera and discussed the current division of the subfamily in 4 tribes. Based on new interrelationships of Allograpta and related taxa found in this article, a molecular study further exploring these placements was recently published by Mengual et al. (2008b).

Morphological characters have used for inferring Syrphidae phylogenies. Rotheray and Gilbert (1989, 1999) scored larval characters for the Palaearctic genera of Syrphidae and presented the first cladistic analysis of the group. Hippa and Ståhls (2005) presented a list of morphological characters with phylogenetic information and their results reported the monophyly of the family and the tribe Pipizini as the sister group of subfamily Syrphinae. Morphological characters have also combined with molecular characters to improve resolution and accuracy. The first combined phylogeny for the family Syrphidae was published by Ståhls et al. (2003), also other authors used DNA and morphological characters for trying to solve systematics of groups within Syrphidae (see Cheng et al. 2000; Ståhls et al. 2004; Milankov et al. 2005, 2007; Masetti et al. 2006; Vujić et al. 2008). 
TABLE 1. Diagnostic characters of Allograpta and related flower flies.

The following is a list of the morphological characters that seem to vary among the distinctive groups related to and included in the genus Allograpta. Although some of these characters are invariant for the included taxa, they will be useful when the syrphines as a whole are analyzed. Characters 7,8 and 26 separate studied taxa from Chrysotoxum; characters 13 and 27 separate them from Ocyptamus; character 24 separate them from Salpingogaster and Lapposyrphus and 3 from Leucopodella.

0 . Face [length], frontal view:

1. short, less than half eye height.

2. medium, about as long as eye height.

3 . long, more than eye height.

1. Face [width], frontal view:

1. narrow, about $1 / 4$ as wide as head.

2. medium, about $1 / 3$ as wide as head.

3. broad, $1 / 2$ or more as wide as head.

2. Face [shape / production], lateral view:

1. straight, not produced anteriorly; oral apex in line with antennal bases (oral opening about 2 times as long as broad) (fig. 8).

2. produced anteriorly; oral apex distinctly more prominent than antennal bases (oral opening 3 or more times longer than broad) (figs. 7, 9 and 10).

3. Facial tubercle:

1. absent (fig. 9).

2. distinct.

4. Facial tubercle [shape]:

1. indistinct or not differentiated dorsally (fig. 8).

2. distinct both dorsally and ventrally (fig. 6).

3. indistinct or not differentiated ventrally (fig. 3).

5. Eye [male]:

1. holoptic.

2. narrowly dichoptic, separated by less than ocellar triangle width.

3 . broadly dichoptic, separated by more than ocellar width.

6. Antennal pits:

1. confluent (fig. 17).

2. distinctly separated (fig. 18).

3. broadly separated (fig. 19).

7. Antenna [length]:

1. short, shorter than facial length.

2. long, longer than facial length.

8. Scape [length]:

1. short, as long as broad or shorter.

2. long, longer than broad.

9. Basoflagellomere [shape / length]:

1. oval, as long as broad.

2. suboval, longer than broad, but not more than 1.5 times as long as broad.

3. elongate, more than 1.5 times as long as broad.

10. Scutum:

1. concolorous laterally and medially.

2. with bright pale (usually yellow) coloration laterally, at least from postpronotum to transverse suture.

11. Anepisternum, anterior flattened portion:

1. bare.

2. with a single row of pile.

3. entirely pilose. 
TABLE 1. (continued)

12. Scutellum [subscutellar fringe]:

1. with complete row of pile on ventral surface.

2. with pile restricted laterally, with median ventral surface bare.

3. completely bare ventrally.

13. Metaepisternum:

1. bare.

2. with tuft or row of pile ventrad to spiracle.

14. Metasternum:

1. bare.

2. pilose.

15. Calypter:

1. bare.

2. ventral lobe pilose on dorsal surface.

16. Plumula:

1. absent or greatly reduced, shorter than subalare (see fig. 130 in Matsuda 1970: 310).

2. short, as long as subalare.

3. long, about 2 times as long as subalare.

17. Metacoxa:

1. with pile posteromedially.

2. bare posteriorly.

18. Wing:

1. microtrichose.

2. bare basomedially, on basal $1 / 3$ or less.

3. bare on basal $2 / 3$ or more.

19. Wing [pattern]:

1. hyaline, without maculae.

2. fasciate, with medial dark fascia.

3. with apical dark macula (fig. 6).

4. with anterior margin dark.

20. Wing [posterior margin]:

1. simple.

2. with minute, discrete, black sclerotized puncta.

21. Vein M1 [curvature]:

1. oblique, slightly sinuate (figs. 1-6).

2. perpendicular, straight or nearly so (see fig. 11 in Vockertoh 1969: 146).

22. Vein M1 [terminal position]:

1. joining vein $R 4+5$ basal to termination of vein $R 2+3$.

2. joining vein $R 4+5$ approximately at level of termination of vein $R 2+3$.

3. joining vein $R 4+5$ apically to termination of vein $R 2+3$.

23. Alula [size]:

1. as broad as or broader than cell BM.

2. narrower than cell BM, but broader than cell $\mathrm{C}$.

3. as broad as or narrower than cell C.

4. absent.

24. Vein R4+5 apically [curvature]:

1. straight or nearly so.

2. sinuate, with a shallow loop into cell R4+5.

3. strongly sinuate, with a large loop into cell R4+5.

25. Abdomen [shape]:

1. oval, broader than thorax.

2. elongate with parallel sides, as wide as thorax.

3. petiolate, basally constricted, apically expanded, always narrower than thorax at constricted area. 
TABLE 1. (continued)

26. Abdomen [shape, cross-section]:

1. flattened.

2. distinctly convex as in Chrysotoxum.

27. Abdomen [length]:

1. short, less than two times as long as thorax (including scutellum).

2. elongate, two times as long as thorax, but less than three times.

3. greatly elongate, three or more times as long as thorax.

28. Abdominal margins:

1. without distinct premarginal sulcus or only weak one on 5th tergum.

2. with distinct premarginal sulcus from 2 nd tergum to 5 th tergum.

29. 5th tergum [shape]:

1. narrow, about two times as broad as long.

2. broad, about as broad as long.

30. Male genitalia [size]:

1. small, with epandrium half as wide as abdomen or less.

2. large, with epandrium about as wide as abdomen.

31. Male genitalia [epandrium postero-apically to cerci]:

1. open, sides of epandrium broadly separated (see fig. 86b in Vockeroth 1969: 165).

2. adjacent, sides of epandrium approximate but with distinct gap between them (see fig. 2a in Carver and Thompson 2003: 38; also see fig. 68 in Doesburg and Doesburg, 1977: 67).

3. closed, with epandrium completely surrounding cerci and without a distinct edge separating the sides (see fig. 87e in Vockeroth 1969: 166).

32. Male genitalia [superior lobe]:

1. fused basally with hypandrium.

2. free and articulated with hypandrium.

33. Male gentialia [aedeagus]:

1. basal aedeagus simple, not swollen nor with denticules.

2. basal aedeagus swollen apically and with black denticules.

3. basal aedeagus complex, without black denticules.

In the present study, a basic matrix of adult morphological characters (Table 1) is given for exemplar species (Table 2). The presumably related groups to Allograpta and the here diagnosed subgroup of Allograpta were included in the analysis. Syrphus ribesii (Linnaeus 1758) was chosen as the outgroup based on the results of Mengual et al. (2008a), and representatives of Episyrphus, Meliscaeva, Anu, Citrogramma, Exallandra, Giluwea, Sphaerophoria and Eosphaerophoria were studied with representatives of the subgenera of Allograpta.

Whether these are useful characters for cladistic analysis depends on a more rigorous examination of their distribution among all syrphine flies and related outgroup taxa and their true homology. As this examination has not been completed, these attributes are only presented as potential sources of useful characters for future phylogenetic analyses.

\section{Allograpta Osten Sacken}

Description (adapted from Vockeroth 1969: 126). Head: Face variable in structure, yellow, with or without medial black vitta; oral opening variable; frons normal, not swollen; antenna short, less than head width; scape about as long as broad, pedicel broader than long; basoflagellomere oval to slightly elongate, not more than 1.6 times as long as broad; arista basal, bare; eye bare; male usually holoptic (only 2 New Zealand species narrowly dichoptic), ommatidia uniform. 
Thorax: Postpronotum bare, yellow; scutum usually yellow laterally to transverse suture or beyond; postalar callus yellow; scutellum at least partially yellow, with distinct ventral pile fringe; anepisternum bare on anterior flattened portion; katepisternum without anterodorsal pile tuft, with dorsal and ventral pile areas separated or narrowly joined posteriorly, otherwise broadly separated anteriorly; metasternum variable; metathoracic pleuron bare; calypter bare, plumula variable. Legs: simple; metacoxa without pile tuft on posteroventral apical angle. Wing: variable; vein R4+5 straight; vein M1 (apical crossvein) processive, slightly sinuate; posterior margin without black sclerotized puncta; alula variable.

Abdomen: variable; terga without marginal sulcus. Male genitalia: typical syrphine form; cercus elongate oval; surstyle elongate oval to triangular; lingula absent; aedeagus two-segmented, with apical segment flared apically, basal segment oval to elongate, with minute dark dense appressed spicules apically (except Antillus); superior lobe fused to hypandrium, usually triangular.

Etymology: Allograpta is derived from "allo" from the Greek meaning "another kind or strange" (Brown 1956: 80) and "grapta" also from the Greek meaning "marked" (Brown 1956: 379). Thus Allograpta means another kind of marking, clearly referring to the unique abdominal color pattern of the type species.

Allograpta is recognized among the syrphines (subfamily Syrphinae, tribe Syrphini) by a combination of characters: 1) eye bare; 2) anterior anepisternum bare; 3) calypter bare; 4) abdomen without premarginal sulcus; 5) metacoxa bare posteromedially; and 6) metathoracic pleuron bare.

TABLE 2. Taxa (specimens) scored for the matrix.

The specimens used to score the taxa for the attribute matrix are as follows. All specimens, unless otherwise noted, are preserved in the USNM.

Allograpta (Allograpta) alamacula Carver Australia (Holotype, ANIC)

Allograpta (Allograpta) javana (Wiedemann) India (USNM ENT 00036027)

Allograpta (Allograpta) obliqua (Say) USA: Texas (USNM ENT 00036016)

Allograpta (Allograpta) ventralis (Miller) New Zealand (USNM ENT 00035389)

Allograpta (Antillus) ascita (Vockeroth) Dominican Republic (USNM ENT 00036015)

Allograpta (Claraplumula) latifacies (Shannon) Peru (Holotype, USNM)

Allograpta (Costarica) nishida Thompson Costa Rica (Holotype, INBIOCRI003724240)

Allograpta (Costarica) zumbadoi Thompson Costa Rica (Paratype, INBIOCRI002472871)

Allograpta (Fazia) centropogonis Nishida Costa Rica (Paratype, INBIOCRI002538891)

Allograpta (Fazia) decemmaculata (Rondani) Chile (Holotype of bullaephora Shannon, USNM)

Allograpta (Rhinoprosopa) aenea (Hull) Dominican Republic (USNM ENT 00036014)

Allograpta (Rhinoprosopa) flavophylla (Hull) Costa Rica (INBIOCRI003788089)

Anu una Thompson New Zealand (Holotype, NZAC)

Citrogramma henryi Ghorphade Sri Lanka (Holotype, USNM)

Eosphaerophoria n. sp. Vietnam (USNM ENT 00036031)

Episyrphus balteatus (De Geer) Ireland (USNM ENT 00036026)

Exallandra cinctifacies (Speiser) Kenya (USNM ENT 00035918)

Giluwea flavomacula Vockeroth New Guinea (Paratype, USNM ENT 00035915)

Meliscaeva cinctella (Zetterstedt) Denmark (USNM ENT 00029694)

Sphaerophoria (Loveridgeana) beattiei (van Doesburg \& van Doesburg) Saint Helena (Paratype, USNM ENT 00114661)

Sphaerophoria (Sphaerophoria) novaeangliae Johnson USA: New Hampshire (USNM ENT 00036030)

Sphaerophoria (Sphaerophoria) scripta (Linnaeus) Germany (USNM ENT 00036029)

Syrphus ribesii (Linnaeus) Norway (USNM ENT 00036028) 

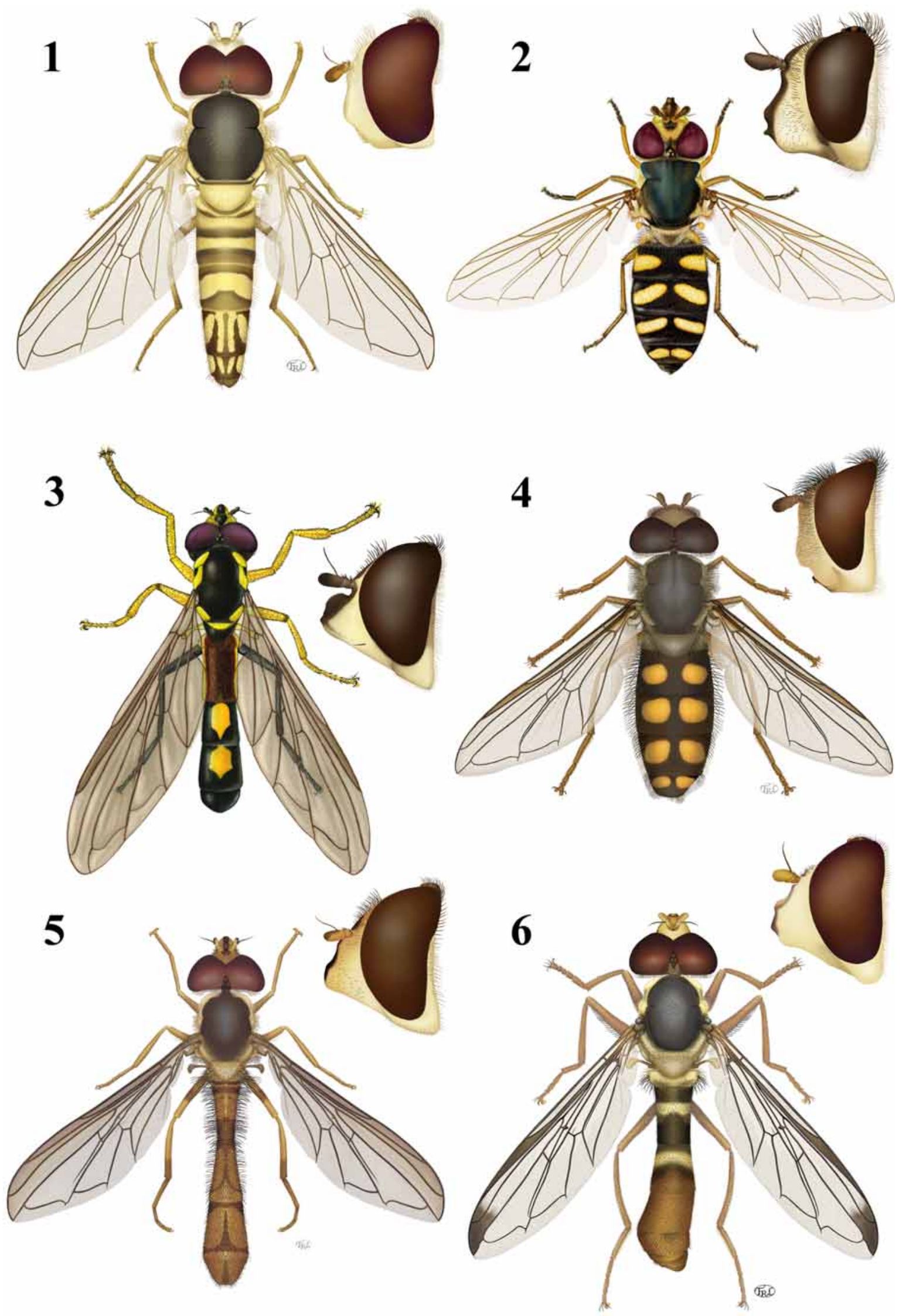

FIGURES 1-6. Habitus, dorsal view, with lateral view of head. 1. Allograpta (Allograpta) obliqua (Say); 2. Allograpta (Fazia) centropogonis Nishida; 3. Allograpta (Costarica) zumbadoi Thompson; 4. Allograpta (Claraplumula) latifacies (Shannon); 5. Allograpta (Rhinoprosopa) aenea (Hull); 6. Allograpta (Antillus) ascitus (Vockeroth). 
The only genera likely to be confused with Allograpta are Sphaerophoria and Exallandra and the confusion is only with the typic subgenus. The other subgenera are readily distinguished from these genera by their facial shape (anteriorly extended) and automorphic characters (apical wing maculae, petiolate abdomen). Vockeroth (1969: 133) pointed out the difficulty of distinguishing Sphaerophoria [and Exallandra] females from those of Allograpta, "specifically if the latter lack the oblique tergite markings commonly found in that genus." Allograpta (sensu stricto) has a complete subscutellar fringe, which is well developed and at least moderately dense, whereas Sphaerophoria and Exallandra have a reduced or no subscutellar fringe.

Distribution. Allograpta is found in all Biotic Regions, but is absent from most of the Palaearctic Region and northern areas of the Nearctic (see fig. 22). The genus is absent from the western Palaearctic and occurs only along the southeastern edge of the region (from southern Far Eastern Russia, south to China). Only the typic subgenus is known from outside the Neotropical Region. The greatest diversity is found in the Neotropics, but there is a small radiation also in New Zealand. The species counts for the various regions are Palaearctic (2), Nearctic (4), Neotropical (52), Afrotropical (12), Oriental (10), Australian (33) and Oceania (10).

Allograpta species are structurally rather uniform throughout the World except in the Neotropics and New Zealand. The structural diversity is documented in the Neotropics by a number of subgeneric groups, but the diversity in New Zealand is not.
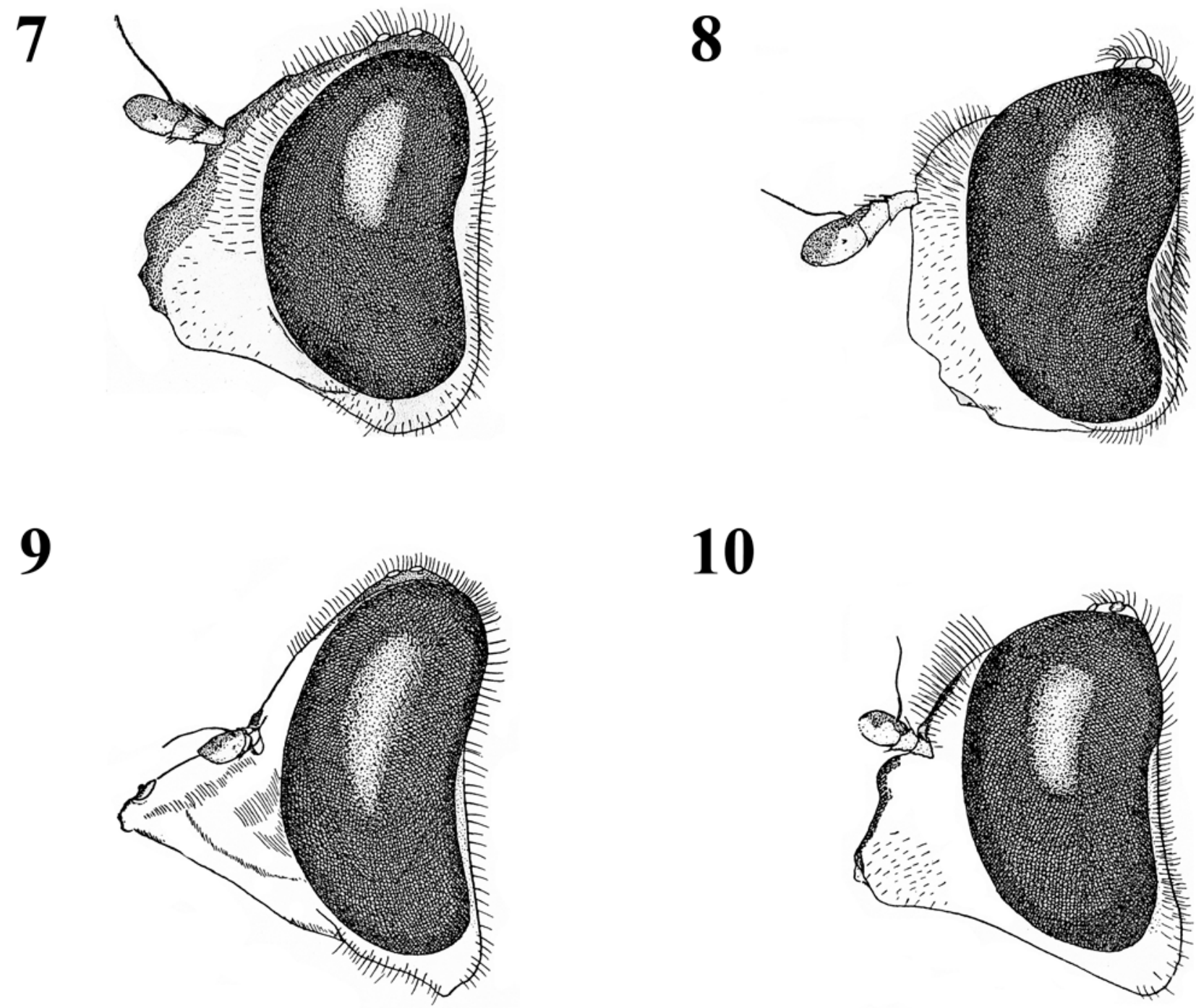

10

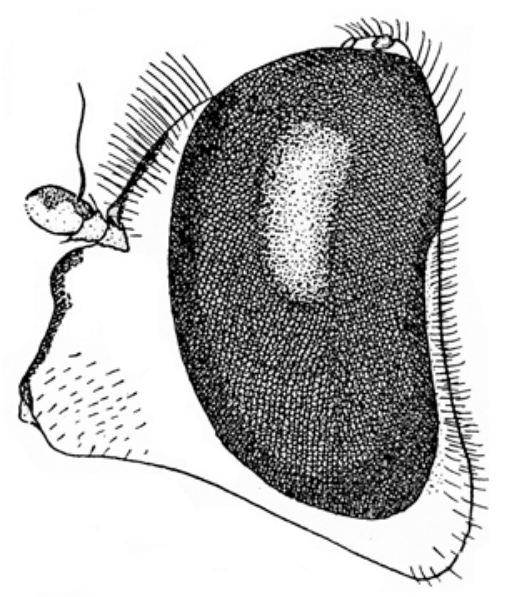

FIGURES 7-10. Heads, lateral view. 7. Allograpta (Fazia) colombia Curran; 8. Allograpta (Allograpta) obliqua (Say); 9. Allograpta (Rhinoprosopa) flavophylla (Hull); 10. Allograpta (Rhinoprosopa) aenea (Hull). 
TABLE 3. Character state matrix for studied taxa.

\begin{tabular}{|c|c|c|c|c|c|c|c|c|c|c|c|c|c|c|c|c|c|c|}
\hline \multirow{2}{*}{ Taxa } & \multicolumn{18}{|c|}{ Characters } \\
\hline & $\mathbf{0}$ & 1 & 2 & 3 & 4 & 5 & 6 & 7 & 8 & 9 & 10 & 11 & 12 & 13 & 14 & 15 & 16 & 17 \\
\hline Allograpta (Allograpta) alamacula & 1 & 2 & 1 & 2 & 2 & 1 & 1 & 1 & 1 & 1 & 2 & 1 & 3 & 1 & 1 & 1 & 1 & 2 \\
\hline Allograpta (Allograpta) javana & 1 & 2 & 1 & 2 & 2 & 1 & 2 & 1 & 1 & 1 & 2 & 1 & 1 & 1 & 2 & 1 & 3 & 2 \\
\hline Allograpta (Allograpta) obliqua & 1 & 1 & 1 & 2 & 1 & 1 & 2 & 1 & 1 & 2 & 2 & 1 & 1 & 1 & 2 & 1 & 3 & 2 \\
\hline Allograpta (Allograpta) ventralis & 1 & 2 & 1 & 2 & 2 & 3 & 1 & 1 & 1 & 1 & 2 & 1 & 3 & 1 & 2 & 1 & 1 & 2 \\
\hline Allograpta (Antillus) ascita & 1 & 2 & 2 & 2 & 2 & 1 & 1 & 1 & 1 & 1 & 2 & 1 & 3 & 1 & 1 & 1 & 1 & 2 \\
\hline Allograpta (Claraplumula) latifacies & 1 & 3 & 1 & 2 & 1 & 1 & 3 & 1 & 1 & 1 & 1 & 1 & 1 & 1 & 2 & 1 & 3 & 2 \\
\hline Allograpta (Costarica) nishida & 1 & 2 & 2 & 2 & 3 & 1 & 3 & 1 & 1 & 1 & 2 & 1 & 3 & 1 & 1 & 1 & 1 & 2 \\
\hline Allograpta (Costarica) zumbadoi & 1 & 2 & 2 & 2 & 3 & 1 & 3 & 1 & 1 & 1 & 2 & 1 & 3 & 1 & 1 & 1 & 1 & 2 \\
\hline Allograpta (Fazia) centropogonis & 2 & 3 & 2 & 2 & 2 & 1 & 3 & 1 & 1 & 1 & 2 & 1 & 1 & 1 & 2 & 1 & 3 & 2 \\
\hline Allograpta (Fazia) decemmaculata & 2 & 3 & 2 & 2 & 2 & 1 & 3 & 1 & 1 & 1 & 2 & 1 & 1 & 1 & 2 & 1 & 3 & 2 \\
\hline Allograpta (Rhinoprosopa) aenea & 1 & 1 & 2 & 2 & 2 & 1 & 3 & 1 & 1 & 1 & 2 & 1 & 3 & 1 & 1 & 1 & 1 & 2 \\
\hline Allograpta (Rhinoprosopa) flavophylla & 1 & 1 & 2 & 1 & 1 & 1 & 3 & 1 & 1 & 1 & 2 & 1 & 3 & 1 & 1 & 1 & 1 & 2 \\
\hline Апи ипа & 1 & 2 & 1 & 2 & 2 & 1 & 1 & 1 & 1 & 1 & 2 & 1 & 2 & 1 & 1 & 1 & 2 & 2 \\
\hline Citrogramma henryi & 2 & 2 & 1 & 2 & 1 & 1 & 2 & 1 & 1 & 2 & 2 & 1 & 1 & 1 & 2 & 1 & 2 & 2 \\
\hline Eosphaerophoria n. sp. & 1 & 1 & 1 & 2 & 2 & 3 & 1 & 1 & 1 & 1 & 2 & 1 & 2 & 1 & 1 & 1 & 1 & 2 \\
\hline Episyrphus balteatus & 1 & 2 & 1 & 2 & 2 & 1 & 1 & 1 & 1 & 1 & 1 & 2 & 1 & 1 & 2 & 1 & 3 & 2 \\
\hline Exallandra cinctifacies & 1 & 2 & 1 & 2 & 2 & 1 & 1 & 1 & 1 & 1 & 2 & 1 & 2 & 1 & 2 & 1 & 2 & 2 \\
\hline Giluwea flavomacula & 1 & 2 & 2 & 2 & 2 & 3 & 2 & 1 & 1 & 1 & 2 & 1 & 1 & 1 & 1 & 1 & 1 & 2 \\
\hline Meliscaeva cinctella & 2 & 2 & 1 & 2 & 2 & 1 & 1 & 1 & 1 & 1 & 1 & 3 & 1 & 1 & 1 & 1 & 3 & 2 \\
\hline Sphaerophoria (Loveridgeana) beattiei & 1 & 1 & 1 & 2 & 1 & 3 & 1 & 1 & 1 & 1 & 2 & 1 & 3 & 1 & 2 & 1 & 2 & 2 \\
\hline $\begin{array}{l}\text { Sphaerophoria (Sphaerophoria) } \\
\text { novaeangliae }\end{array}$ & 1 & 2 & 1 & 2 & 2 & 1 & 1 & 1 & 1 & 1 & 2 & 1 & 2 & 1 & 2 & 1 & 1 & 2 \\
\hline Sphaerophoria (Sphaerophoria) scripta & 1 & 2 & 1 & 2 & 2 & 1 & 1 & 1 & 1 & 2 & 2 & 1 & 2 & 1 & 2 & 1 & 3 & 2 \\
\hline Syrphus ribesii & 2 & 2 & 1 & 2 & 2 & 1 & 1 & 1 & 1 & 2 & 1 & 1 & 1 & 1 & 1 & 2 & 3 & 1 \\
\hline
\end{tabular}

continued.

\begin{tabular}{|c|c|c|c|c|c|c|c|c|c|c|c|c|c|c|c|c|}
\hline \multirow{2}{*}{ Taxa } & \multicolumn{16}{|c|}{ Characters } \\
\hline & 18 & 19 & 20 & 21 & 22 & 23 & 24 & 25 & 26 & 27 & 28 & 29 & 30 & 31 & 32 & 33 \\
\hline Allograpta (Allograpta) alamacula & 2 & 3 & 1 & 1 & 2 & 1 & 1 & 2 & 1 & 2 & 1 & 1 & 1 & 2 & 1 & 2 \\
\hline Allograpta (Allograpta) javana & 2 & 1 & 1 & 1 & 3 & 1 & 1 & 2 & 1 & 2 & 1 & 1 & 1 & 1 & 1 & 2 \\
\hline Allograpta (Allograpta) obliqua & 3 & 1 & 1 & 1 & 2 & 1 & 1 & 2 & 1 & 2 & 1 & 1 & 1 & 1 & 1 & 2 \\
\hline Allograpta (Allograpta) ventralis & 2 & 1 & 1 & 1 & 2 & 3 & 1 & 3 & 1 & 2 & 1 & 1 & 1 & 1 & 1 & 2 \\
\hline Allograpta (Antillus) ascita & 3 & 3 & 1 & 1 & 2 & 1 & 1 & 2 & 1 & 2 & 1 & 1 & 2 & 1 & 1 & 3 \\
\hline Allograpta (Claraplumula) latifacies & 2 & 1 & 1 & 1 & 1 & 1 & 1 & 1 & 1 & 1 & 1 & 1 & 1 & 1 & 1 & 2 \\
\hline Allograpta (Costarica) nishida & 1 & 1 & 1 & 1 & 1 & 2 & 1 & 2 & 1 & 2 & 1 & 2 & 1 & 1 & 1 & 2 \\
\hline Allograpta (Costarica) zumbadoi & 1 & 1 & 1 & 1 & 1 & 2 & 1 & 2 & 1 & 2 & 1 & 2 & 1 & 1 & 1 & 2 \\
\hline Allograpta (Fazia) centropogonis & 2 & 1 & 1 & 1 & 2 & 1 & 1 & 2 & 1 & 1 & 1 & 1 & 1 & 1 & 1 & 2 \\
\hline Allograpta (Fazia) decemmaculata & 2 & 1 & 1 & 1 & 1 & 1 & 1 & 2 & 1 & 1 & 1 & 1 & 1 & 1 & 1 & 2 \\
\hline Allograpta (Rhinoprosopa) aenea & 1 & 1 & 1 & 1 & 2 & 3 & 1 & 3 & 1 & 3 & 1 & 2 & 1 & 1 & 1 & 2 \\
\hline Allograpta (Rhinoprosopa) flavophylla & 1 & 1 & 1 & 1 & 2 & 3 & 1 & 3 & 1 & 3 & 1 & 2 & 1 & 1 & 1 & 2 \\
\hline Anu una & 2 & 1 & 1 & 1 & 3 & 2 & 1 & 2 & 1 & 1 & 1 & 1 & 1 & 1 & 2 & 1 \\
\hline
\end{tabular}




\begin{tabular}{|c|c|c|c|c|c|c|c|c|c|c|c|c|c|c|c|c|}
\hline \multirow{2}{*}{ Taxa } & \multicolumn{16}{|c|}{ Characters } \\
\hline & $\mathbf{0}$ & $\mathbf{1}$ & 2 & 3 & 4 & 5 & 6 & 7 & 8 & 9 & 10 & 11 & 12 & 13 & 14 & 15 \\
\hline Episyrphus balteatus & 2 & 1 & 2 & 1 & 3 & 1 & 1 & 2 & 1 & 1 & 1 & 1 & 1 & 1 & 2 & 1 \\
\hline Exallandra cinctifacies & 2 & 1 & 1 & 1 & 3 & 1 & 1 & 2 & 1 & 1 & 1 & 1 & 1 & 1 & 2 & 1 \\
\hline Giluwea flavomacula & 1 & 1 & 1 & 1 & 3 & 3 & 1 & 2 & 1 & 1 & 1 & 1 & 1 & 1 & 2 & 1 \\
\hline Meliscaeva cinctella & 2 & 1 & 2 & 1 & 3 & 1 & 1 & 2 & 1 & 1 & 1 & 1 & 1 & 1 & 2 & 1 \\
\hline Sphaerophoria (Loveridgeana) beattiei & 1 & 1 & 1 & 1 & 3 & 2 & 1 & 2 & 1 & 2 & 1 & 2 & 2 & 2 & 2 & 1 \\
\hline $\begin{array}{l}\text { Sphaerophoria (Sphaerophoria) } \\
\text { novaeangliae }\end{array}$ & 2 & 1 & 1 & 1 & 3 & 2 & 1 & 2 & 1 & 2 & 1 & 1 & 2 & 1 & 2 & 1 \\
\hline Sphaerophoria (Sphaerophoria) scripta & 2 & 1 & 1 & 1 & 3 & 1 & 1 & 2 & 1 & 3 & 1 & 2 & 2 & 3 & 2 & 1 \\
\hline Syrphus ribesii & 2 & 1 & 1 & 1 & 2 & 1 & 1 & 1 & 1 & 1 & 2 & 1 & 1 & 1 & 2 & 1 \\
\hline
\end{tabular}

\section{Key to the subgenera and species groups of Allograpta}

1. Metasternum pilose; plumula present and well developed .................................................................................... 6

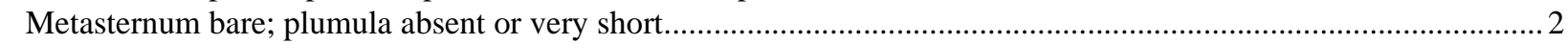

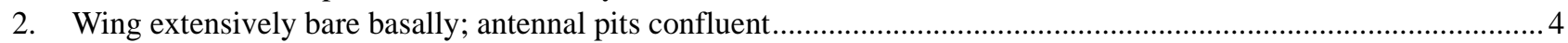

- Wing completely microtrichose; antennal pits separate. Alula narrow, narrower than cell BM ................................. 3

3. Face with abrupt tubercle; abdomen parallel-sided (fig. 3) ......................................................................... Costarica

- Face with indistinct or no tubercle (fig. 9-10); abdomen petiolate (fig. 5) .............................................Rhinoprosopa

4. Alula narrow, as wide as costal cell; male broadly dichoptic; wing without distinct apical macula.......

Allograpta, ventralis group

- Alula broad, broader than costal cell, as broad or broader than cell BM; male holoptic; wing with distinct apical brown macula

5. Face produced anteriorly; oral apex distinctly more prominent than antennal bases; oral opening 3.5 times longer than broad (fig. 6); large flies, $13 \mathrm{~mm}$ or greater. Antillus

- Face straight, not produced anteriorly; oral apex distinctly less prominent than antennal bases; oral opening only about 2 times as long as broad; small flies, $6 \mathrm{~mm}$ or less. Allograpta, alamacula group

6. Face produced forward; oral apex distinctly more prominent than antennal bases; oral opening 3 or more times longer than broad; facial tubercle high, distinctly differentiated (fig. 7)..... Fazia

- $\quad$ Face straight, not produced forward; oral apex distinctly less prominent than antennal bases; oral opening only about 2 times as long as broad; facial tubercle usually low (except armillata), not differentiated dorsally (figs. 1, 8) ........ 7

7. Abdomen oval, with 4 pairs of large round maculae (fig. 4); large flies, $13 \mathrm{~mm}$.. Claraplumula

- Abdomen elongate, tapering, usually with fasciae and/or vittae (fig. 1); smaller flies, usually $10 \mathrm{~mm}$ or less .... Allograpta, obliqua group

\section{Subgenus Allograpta Osten Sacken}

Allograpta Osten Sacken 1875: 49. Type-species, Scaeva obliqua Say (monotypy).

Neoscaeva Frey 1946: 170. Type-species, Syrphus aeruginosifrons Schiner (original designation).

Microsphaerophoria Frey 1946: 168. Type-species, plaumanni Frey (original designation).

Miogramma Frey 1946: 165. Type-species, Syrphus javanus Wiedemann (original designation).

Helenomyia Bankowska 1962: 311. Type-species, Syrphus javanus Wiedemann (original designation).

Paraxanthogramma Tao \& Chiu 1971: 74 nomen nudum based on Syrphus javanus Wiedemann as nakamurae Matsumura.

Diagnosis. Face straight, with low indistinct tubercle of variable size; oral opening about 1.5 to 2 times as long as wide, with oral apex at level of antennal base; antennal pits confluent; plumula well developed or absent; subscutellar fringe distinct or absent; wing partially bare basomedially, with or without apical dark macula; alula broad, about 1.5 times as broad as cell BM; metasternum pilose or bare; abdomen elongate, parallel to slightly petiolate. 

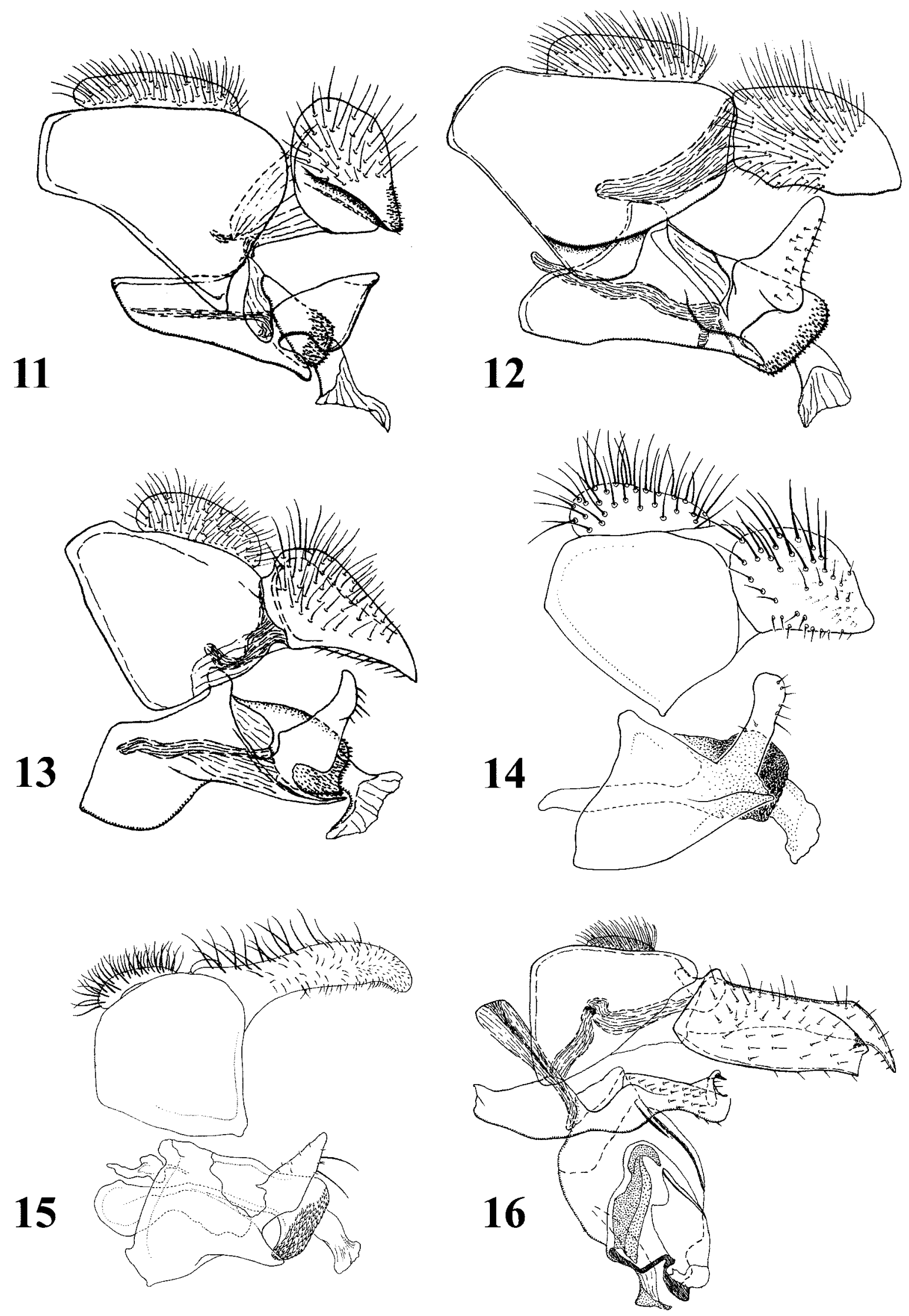

FIGURES 11-16. Male genitalia, lateral view. 11. Allograpta (Allograpta) obliqua (Say); 12. Allograpta (Claraplumula) latifacies (Shannon); 13. Allograpta (Rhinoprosopa) aenea (Hull); 14. Allograpta (Costarica) zumbadoi Thompson; 15. Allograpta (Fazia) centropogonis Nishida; 16. Allograpta (Antillus) ascitus (Vockeroth). 

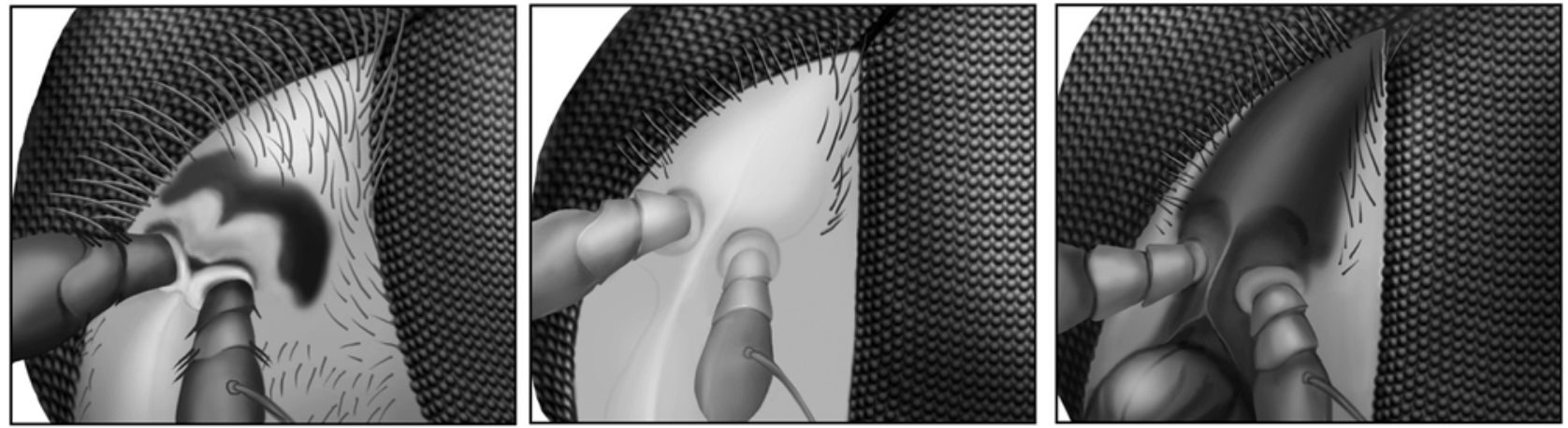

FIGURES 17-19. Heads, oblique anterodorsal view of antennal pits. 17. Syrphus ribesii Linnaeus; 18. Allograpta (Allograpta) obliqua (Say); 19. Allograpta (Costarica) zumbadoi Thompson.

\section{Allograpta obliqua species group}

Diagnosis. Face straight, with low indistinct tubercle; oral opening about 1.5 times as long as wide, with oral apex at level of antennal base; antennal pits confluent; plumula well developed; subscutellar fringe distinct; wing partially bare basomedially, without apical dark macula; alula broad, about 1.5 times as broad as cell BM; metasternum pilose; abdomen elongate.

Included species. AFROTROPICAL: borbonica Kassebeer 2000: 46 (Allograpta) Reunion; **calopoides Curran 1938: 9 (Syrphus) Kenya, Ethiopia, Tanzania, Uganda, Congo; *calopus Loew 1858: 379 (Syrphus) Sudan, Ethiopia, Kenya, Namibia, Nigeria [=abyssinica Frey 1946: 168 (Miogramma)]; *fuscotibialis Macquart 1842: 155 (Syrphus) South Africa [=rotundicornis Loew 1858: 379 (Syrphus)]; hypoxantha Bezzi 1923: 343 (Syrphus) Kenya, Madagascar, Tanzania, Uganda, Congo [=calinus Curran 1938: 10 (Syrphus)]; nasuta Macquart 1842: 156 (Syrphus) western Africa, Diego Garcia, Mauritius, Reunion, Madagascar [=pfeifferi Bigot 1884: 89 (Syrphus)]; nigra Keiser 1971: 225 (Epistrophe) Madagascar; nummularia Bezzi 1920: 137 (Syrphus) Tanzania, Kenya; phaeoptera Bezzi 1920: 139 (Xanthogramma) Tanzania; rediviva Bezzi 1915: 34 (Syrphus) South Africa; rufifacies Keiser 1971: 225 (Epistrophe) Madagascar; tenella Keiser 1971: 227 (Epistrophe) Madagascar; **varipes Curran 1927b: 55 (Epistrophe) Congo, Uganda.

AUSTRALIAN: **atkinsoni Miller 1921: 311 (Platycheirus) New Zealand; *australensis Schiner 1868: 347 (Melithreptus) Australia (NSW, Q1d.); buruensis Meijere 1929: 383 (Allograpta) Indonesia (Buru); *distincta Kertesz 1899: 177 (Melithreptus), New Guinea to Fiji \& Solomons; **flavofaciens Miller 1921: 302 (Syrphus) New Zealand; **hirsutifera Hull 1949d: 727 (Epistrophe) New Zealand; **hudsoni Miller 1921: 302 (Syrphus) New Zealand; pallida Bigot 1884: 93 (Syrphus) Australia; **pseudoropalus Miller 1921: 293 (Paragus) New Zealand; *ropalus Walker 1849: 593 (Syrphus) New Zealand.

NEARCTIC: **exotica Wiedemann 1830: 136 (Syrphus) Oregon to North Carolina, s. to Argentina, Hawaii (introduced) [=quadrigemina Thomson 1869: 500 (Syrphus), =fracta Osten Sacken 1877: 331 (Allograpta), =bilineata Enderlein 1938a: 220 (Allograpta), =duplofasciata Enderlein 1938a: 217 (Allograpta)]; *obliqua Say 1823: 89 (Scaeva) Washington to Quebec, s. to Argentina; Hawaii (introduced) [=securiferus Macquart 1842: 100 (Syrphus), =baccides Walker 1849: 594 (Syrphus), =dimemsus Walker 1852: 235 (Syrphus), =signatus Wulp 1867: 144 (Syrphus), =dejongi Doesburg 1958: 44 (Allograpta)]; **radiata Bigot (see below) Florida.

NEOTROPICAL: **aeruginosifrons Schiner 1868: 352 (Syrphus) Brazil; annulipes Macquart 1850: 464 (Paragus) Colombia, Ecuador, Peru, Brazil, Bolivia [=scutellata Sack 1941: 99 (Allograpta), =geminata Fluke 1942: 16 (Allograpta)]; **aperta Fluke 1942: 19 (Allograpta) Surinam, Guyana [=notata Doesburg 
1966: 64 (Allograpta)]; **bilineella Enderlein 1938a: 219 (Allograpta) Colombia; **browni Fluke 1942: 17 (Allograpta) Ecuador; **exotica Wiedemann (see above); **falcata Fluke 1942: 16 (Allograpta) Colombia, Venezuela, Ecuador, Peru; **hastata Fluke 1942: 17 (Allograpta) Peru, Brazil; *hortensis Philippi 1865: 746 (Syrphus) Peru, Chile, Argentina [=bimaculata Enderlein 1938a: 223 (Allograpta)]; **insularis Thompson 1981: 28 (Allograpta) Puerto Rico; **limbata Fabricius 1805: 251 (Scaeva) West Indies, Brazil [=fuscisquama Curran 1927a: 4 (Allograpta)]; **neotropica Curran 1936: 14 (Allograpta) Colombia, Ecuador, Brazil, Argentina); *obliqua Say (see above); **piurana Shannon 1927: 9 (Allograpta) Peru, Chile [=chilensis Sack 1941: 98 (Allograpta), =harlequina Hull 1949c: 76 (Allograpta)]; **pulchra Shannon 1927: 25 (Allograpta) Chile; Easter Island [=bifasciata Enderlein 1938a: 221 (Allograpta)]; *radiata Bigot 1857: 338 (Syrphus) Florida, West Indies, intr. Hawaii (not established) [=venusta Curran 1927a: 5 (Allograpta), =cubana Curran 1932: 3 (Allograpta)]; *robinsoniana Enderlein 1938b: 664 (Allograpta) Juan Fernandez Island; *splendens Thomson 1869: 501 (Syrphus) Galápagos; **tectiforma Fluke 1942: 18 (Allograpta) Ecuador; **teligera Fluke 1942: 18 (Allograpta) Ecuador, Brazil; **trilimbata Bigot 1889: 253 (Sphaerophoria) Mexico.

OCEANIAN: *amphoterum Bezzi 1928: 74 (Xanthogramma) Rarotonga, Fiji; **citronella Shiraki 1963: 136 (Epistrophe) Caroline Is.: Palau \& Ponape; *distincta Kertesz (see above); **longulus Shiraki 1963: 139 (Epistrophe) Guam; *nigripilosa Hull 1944a: 52 (Xanthogramma) Society Is.; **neofasciata Thompson 1989: 441 Marianas Is. [=fasciata Shiraki 1963: 139 (Epistrophe); *obliqua Say (see above, introduced Hawaii); *radiata Bigot 1857: 338 (see above, intr. Hawaii (not established) **septemvittata Shiraki 1963: 138 (Epistrophe) Mariana Is.

ORIENTAL: **dravida Ghorpade 1994: 7 (Allograpta) India; *javana Wiedemann 1824: 34 (Syrphus) India to Japan, s. Indonesia [= nakamurae Matsumura 1918: 9 (Xanthogramma)]; *kinabalensis Curran 1931: 350 (Syrphus) Borneo; *maculipleura Brunetti 1913: 162 (Syrphus) India, Myanmar [=bouvieri Herve-Bazin 1923: 26 (Xanthogramma)]; medanensis Meijere 1914: 166 (Sphaerophoria) Sumatra; obscuricornis Meijere 1914: 165 (Sphaerophoria) Java; philippina Frey 1946: 167 (Miogramma) Phillipines; purpureicollis Frey 1946: 167 (Miogramma) Sumatra; *robinsoni Curran 1928: 208 (Syrphus) Malaya [=nigrotibialis Curran 1928: 241 (Sphaerophoria)].

PALAEARCTIC: *javana Wiedemann (see above) Japan; maritima Mutin 1986: 829 (Allograpta) Russian Far East.

\section{Allograpta alamacula species group}

Diagnosis. Face straight, with large tubercle; oral opening about 2 times as long as wide, with oral apex at level of antennal base; antennal pits confluent; plumula absent; subscutellar pile fringe absent; wing broadly bare basomedially, with apical dark macula; alula broad, as broad as cell BM; metasternum bare; abdomen elongate.

Included species. AUSTRALIAN: **alamacula Carver 2003: 37 (Allograpta) Australia (Queensland).

Carver (Carver \& Thompson 2003) reared her species from maggots preying on whiteflies (Aleurocanthus $t$-signatus (Aleyrodidae)). Also see Carver and Thompson (2003: 38, figs. 1 and 2) for black and white habitus and male genitalia.

\section{Allograpta ventralis species group}

Diagnosis. Face straight, with large low tubercle; oral opening about 2 times as long as wide, with oral apex at level of antennal base; antennal pits confluent; plumula absent; subscutellar pile fringe absent; wing broadly bare basomedially, without apical dark macula; alula narrow, about 1/2 as broad as cell BM; metasternum bare or with 2-3 very short pile; abdomen elongate or slightly petiolate. 
Included species. AUSTRALIAN: **dorsalis Miller 1924: 284 (Ocyptamus) New Zealand; **ventralis Miller 1921: 296 (Sphaerophoria) New Zealand.

The biology of ventralis was described by Bowie (2001) and the species are typical Allograpta predators which feed on mealybugs (Balanococcus cordylinidis (Pseudococcidae)). See also Thompson (2008: 4, fig. 4) for colour habitus.

\section{Subgenus Antillus Vockeroth}

Antillus Vockeroth 1969: 130. Type-species, ascitus Vockeroth (original designation).

Diagnosis. Face greatly produced anteriorly, with low small tubercle; oral opening about 3.5 times as long as wide, with oral apex greatly extended beyond level of antennal base; antennal pits confluent; plumula absent; subscutellar pile fringe absent; wing broadly bare basomedially, with apical dark macula; alula broad, much broader than cell BM; metasternum bare; abdomen elongate.

Included species. NEOTROPICAL: **ascita Vockeroth 1969: 130 (Antillus) Hispaniola.

\section{Subgenus Claraplumula Shannon}

Claraplumula Shannon 1927: 8. Type-species, latifacies Shannon (original designation).

Diagnosis. Face straight, with tubercle; oral opening about twice as long as wide, with oral apex at level of antennal base; antennal pits broadly separated; plumula well developed, long; subscutellar pile fringe abundant, dense; wing extensively microtrichose, only narrowly bare basally, without apical dark macula; alula broad, about 1.5 times as broad as cell BM; metasternum pilose; abdomen oval.

Included species. NEOTROPICAL: **latifacies Shannon 1927: 8 (Claraplumula) Ecuador, Peru.

\section{Subgenus Fazia Shannon}

Fazia Shannon 1927: 25. Type-species, bullaephora Shannon (original designation) = decemmaculata (Rondani). Chasmia Enderlein 1938a: 213 (preoccupied by Enderlein 1922). Type-species, hians Enderlein (original designation). Metepistrophe Hull 1949b: 293 (as subgenus of Epistrophe). Type-species, Epistrophe altissima Fluke (original designation). Misidentified type-species. Originally named type was Epistrophe remigis Fluke, but Fluke (1951) noted that the specimen used by Hull was mislabelled by him and was actually altissima Fluke. Hence, under Article 70.3 of the International Code of Zoological Nomenclature (ICZN 1999), we hereby fixed the type species of Metepistrophe Hull to the taxonomical species, altissima Fluke.

Metallograpta Hull 1949b: 293 (as subgenus of Epistrophe). Type-species, Allograpta colombia Curran (original designation).

Diagnosis. Face moderately to greatly produced anteriorly, with distinct tubercle; oral opening about 5 times as long as wide, with oral apex greatly extended beyond level of antennal base; antennal pits separate; plumula well developed; subscutellar pile fringe distinct, one or two rows of long pili; wing broadly bare basomedially, without apical dark macula; alula broad, about 1.5 times as broad as cell BM; metasternum pilose; abdomen broadly to narrowly elongate.

Included species. NEARCTIC: **micrura Osten Sacken 1877: 330 (Sphaerophoria) NE: British Columbia, south to California, Texas \& Mexico [=picticauda Bigot 1884: 102 (Sphaerophoria), =trifasciata Enderlein 1938a: 218 (Allograpta), =transversa Hull 1943a: 32 (Sphaerophoria)].

NEOTROPICAL: **alta Curran 1936: 15 (Allograpta) Colombia, Ecuador [=flavomaculata Hull 1937b: 169 (Allograpta)]; **altissima Fluke 1942: 10 (Epistrophe) Ecuador; **argentipila Fluke 1942: 13 
(Epistrophe) Ecuador, Peru, Argentina; **centropogonis Nishida in Nishida et al. 2002: 423 (Allograpta) Costa Rica; **colombia Curran 1925a: 349 (Allograpta) Colombia, Ecuador, Brazil, Argentina; *decemmaculata Rondani 1863: 12 (Syrphus) Chile, Ecuador [=bullaephora Shannon 1927: 25 (Fazia)]; **eupeltata Bigot 1884: 91 (Syrphus) Mexico [=mexicana Enderlein 1938a: 212 (Fazia)]; **fasciata Curran 1932: 4 (Allograpta) Ecuador, Peru [=bisinterrupta Enderlein 1938a: 210 (Fazia)]; **fascifrons Macquart 1846: 265 (Syrphus) Colombia, Ecuador [=armillata Fluke 1942: 12 (Epistrophe)]; **flukei Curran 1936: 13 (Allograpta) Cuba; **funeralia Hull 1944b: 27 (Epistrophe) NT: Jamaica; **hians Enderlein 1938a: 213 (Chasmia) Mexico; *imitator Curran 1925a: 351 (Epistrophe) Colombia, Ecuador, Bolivia; **luna Fluke 1942: 8 (Epistrophe) Ecuador; *macquarti Blanchard 1852: 411 (Syrphus) Chile, Argentina [=australis Shannon 1927: 26 (Fazia)]; $\boldsymbol{m u}$ Bigot 1884: 105 (Mesograpta) Mexico; **nasigera Enderlein 1938a: 212 (Fazia) Colombia; **plaumanni Frey 1946: 168 (Microsphaerophoria) Brazil; **remigis Fluke 1942: 9 (Epistrophe) Ecuador; **roburoris Fluke 1942: 11 (Epistrophe) Ecuador, Argentina; **rostrata Bigot 1884: 102 (Sphaerophoria) Mexico [=nasuta Enderlein 1938a: 214 (Chasmia), =rhina Thompson in Thompson et al. 1976: 37 (Allograpta)]; **saussurii Giglio-Tos 1892: 2 (Syrphus) Mexico; **similis Curran 1925a: 350 (Allograpta) Colombia, Brazil, Argentina; **strigifacies Enderlein 1938a: 211 (Fazia) Brazil [=hermosa Hull 1941: 48 (Allograpta), =brunneola Frey 1946: 170 (Neoscaeva)]; **syrphica Giglio-Tos 1892: 2 (Sphaerophoria) Mexico; **willistoni Giglio-Tos 1893: 31 (Sphaerophoria) Mexico [=forreri Giglio-Tos 1893: 32 (Sphaerophoria)].

\section{Subgenus Rhinoprosopa Hull}

Oligorhina Hull 1937a: 30 (preoccupied by Fairmaire \& Germain 1863). Type-species, aenea Hull (original designation).

Rhinoprosopa Hull 1942: 23 (new name for Oligorhina Hull).

Diagnosis. Face greatly produced anteriorly, with low indistinct tubercle or no tubercle; oral opening about 5.5 times as long as wide, with oral apex greatly extended beyond level of antennal base; antennal pits distinctly separated; plumula absent; subscutellar pile fringe absent; wing microtrichose, without apical dark macula; alula absent or narrow, less than $1 / 2$ as wide as cell BM; metasternum bare; abdomen narrowly to strongly petiolate.

Included species. NEOTROPICAL: **aenea Hull 1937a: 31 (Oligorhina) Hispaniola; **flavophylla Hull 1943b: 139 (Rhinoprosopa) Peru; **lucifera Hull 1943c: 216 (Rhinoprosopa) Ecuador; **nasuta Bigot 1884: 103 (Sphaerophoria) Mexico [=nasuta Bigot 1888: 253 (Sphaerophoria)]; **sycorax Hull 1947: 239 (Rhinoprosopa) Costa Rica, Colombia, Venezuela.

\section{Subgenus Costarica Mengual \& Thompson, subgen. nov.}

Type-species: Allograpta zumbadoi Thompson

Diagnosis. Face greatly produced anteriorly, with no tubercle but distinctly concave beneath antenna creating an appearance of a tubercle; oral opening about 5 times as long as wide, with oral apex greatly extended beyond level of antennal base; antennal pits distinctly separated; plumula absent; subscutellar fringe absent; wing microtrichose, without apical dark macula; metasternum bare; abdomen narrowly petiolate or elongate.

Included species. NEOTROPICAL: **nishida Mengual \& Thompson, sp. nov. Costa Rica; **zumbadoi Thompson 2000: 34 (Allograpta) Costa Rica.

Costarica is readily distinguished from all other Allograpta groups by the distinctive facial shape and by the dense thick appressed pile on 1 st and 2 nd terga in the males. These characters are unique among flower flies. 
The life history of the type species, zumbadoi, has been worked out by Zuijen and Nishida (2009). The larval stages are stem-borers in Centropogon (Campanulaceae). The plant genus ranges from Mexico to Peru and contains some 230 species, but so far these flies are only known from the higher elevations in Costa Rica.

Etymology. The name Costarica is the name of the country which is the home for the two included species and is to be treated as feminine.

\section{Key to the species of Allograpta (Costarica)}

1. Male \& female with medial yellow macula on 3rd \& 4th terga; female 2nd tergum entirely yellow; pro and mesofemora extensively yellow; katepisternum, postalar callus yellow ..................................................... zumbadoi

- Male \& female with basal arcuate yellow fasciae on 3rd \& 4th terga; female 2nd tergum black with submedial fasciate yellow macula; pro and mesofemora extensively black; katepisternum, postalar callus brownish-black.... nishida

\section{Allograpta (Costarica) nishida Mengual \& Thompson, sp. nov.}

Figures 20 and 21.

Male. Head: Face black medially, narrowly yellow laterally, short yellow pilose laterally; gena yellow, yellow pilose; lunule black; frontal triangle black except very narrowly yellow laterally, black pollinose and pilose; eye contiguity long, as long as vertical triangle; vertical triangle black, black pollinose and pilose; occiput yellow on ventral $1 / 3$ and black on dorsal $2 / 3$, white pollinose and yellow pilose on ventral $2 / 3$, black pollinose and pilose on dorsal 1/3. Antenna brownish black except pale yellowish orange basoventral 1/3 of basoflagellomere, black pilose; arista black.

Thorax: Black except yellow posterior notopleuron, narrowly along base of scutellum, posterior 1/3 of anepisternum; yellow pilose except black pilose on dorsolaterally to wing base; calypter black; halter black except yellow capitulum. Legs: brownish black except yellow femoral apices and basal 1/3 of tibiae, black pilose except a few yellow pili on bases of femora. Wing: hyaline except costal and subcostal darker brownish, microtrichose.

Abdomen: Elongate, slightly narrower than thorax, shiny brownish black except for basal arcuate yellow fasciae on 3rd-4th terga and yellow lateral $1 / 3$ of 1 st tergum, black pilose except long lateral white pile and with dense thick brownish-black pile at dorsomedial part on 1st and 2nd terga (fig. 20a); sterna dark; 1st sternum long white pilose; 2 nd sternum short white pilose; 3rd-5th sterna appressed black pilose. Male genitalia as figured (fig. 21).

Female. Similar to male except for normal sexual dimorphism and: frons black except yellow laterally on ventral $2 / 3$, dull, yellow pilose; 2 nd tergum with arcuate yellow fascia narrowly divided medially. Abdomen mainly shiny, dull black pollinose on most of 2nd terga except apical margin and yellow fasciae, black pollinose areas on 3rd thru 5th terga restricted to medial area posterior to yellow fasciae (see fig. 20b).

Types. Holotype: Male from Costa Rica, Limon: Parque Internacional La Amistad, sendero Circular, 2406 m, LS 340258 577465, 20 June - 5 July 2003, D. Rubi, net collected, \#74159 (1 § INB0003724240 INBIO), deposited in Instituto Nacional de Biodiversidad, Santo Domingo. Paratypes: COSTA RICA. Cartago: Genesis II, 2360 m, LN 188600 545900, 9 June 1994, M. M. Chavarria, \#3157 (1 \& INBIOCRI002014790 USNM); Puntarenas, Parque Internacional La Amistad, Cerro Hoffman, Sendero hacia, 2160 m, LS 337931 575490, 30 June-5 July 2003, D. Rubi, net collected, \#74163, (1 9 INB0003724104, USNM)

Length. Male (1): body, $11.3 \mathrm{~mm}$; wing, $10.0 \mathrm{~mm}$. Female (2): body, 11.5-13.0 (12.3) mm; wing, 10.111.8 (10.9) $\mathrm{mm}$.

Etymology. This species is named after Kenji Nishida in recognition of his discoveries on the diverse life histories of Allograpta species in Costa Rica. The name is to be treated as a noun in apposition.

Allograpta nishida is readily distinguished from zumbadoi as outlined in the key. 


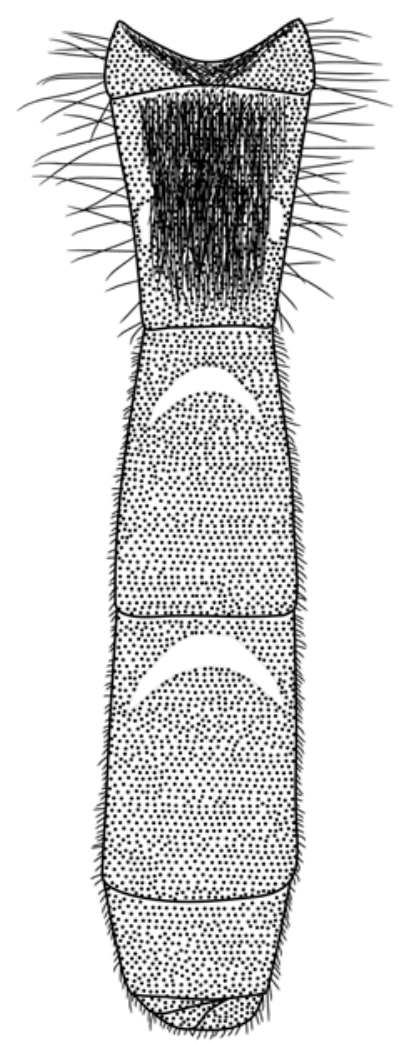

20 a

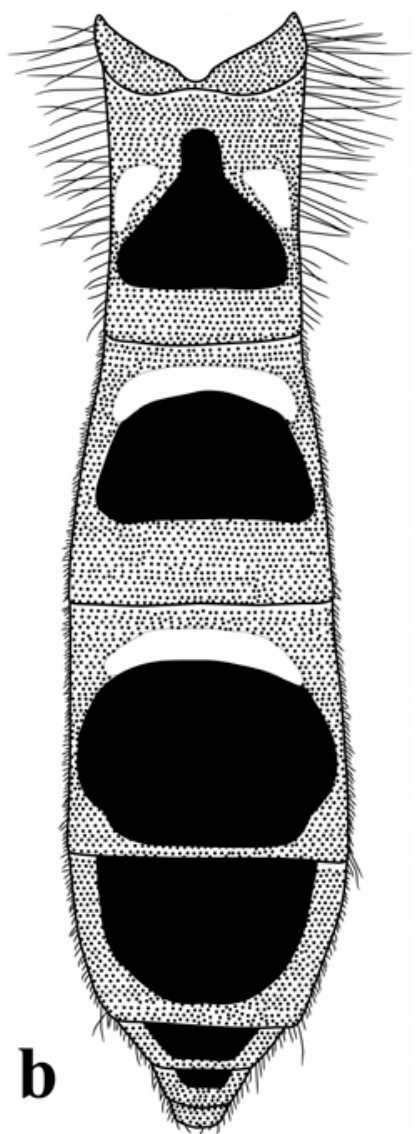

FIGURE 20. Abdomen of Allograpta (Costarica) nishida sp. nov., dorsal view: a, male; b, female.

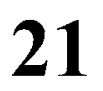

21
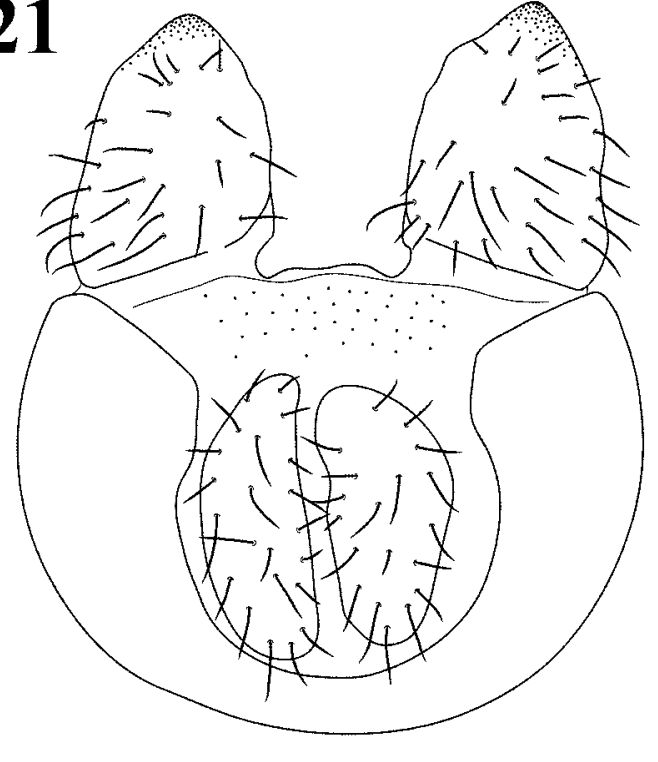

$0.5 \mathrm{~mm}$

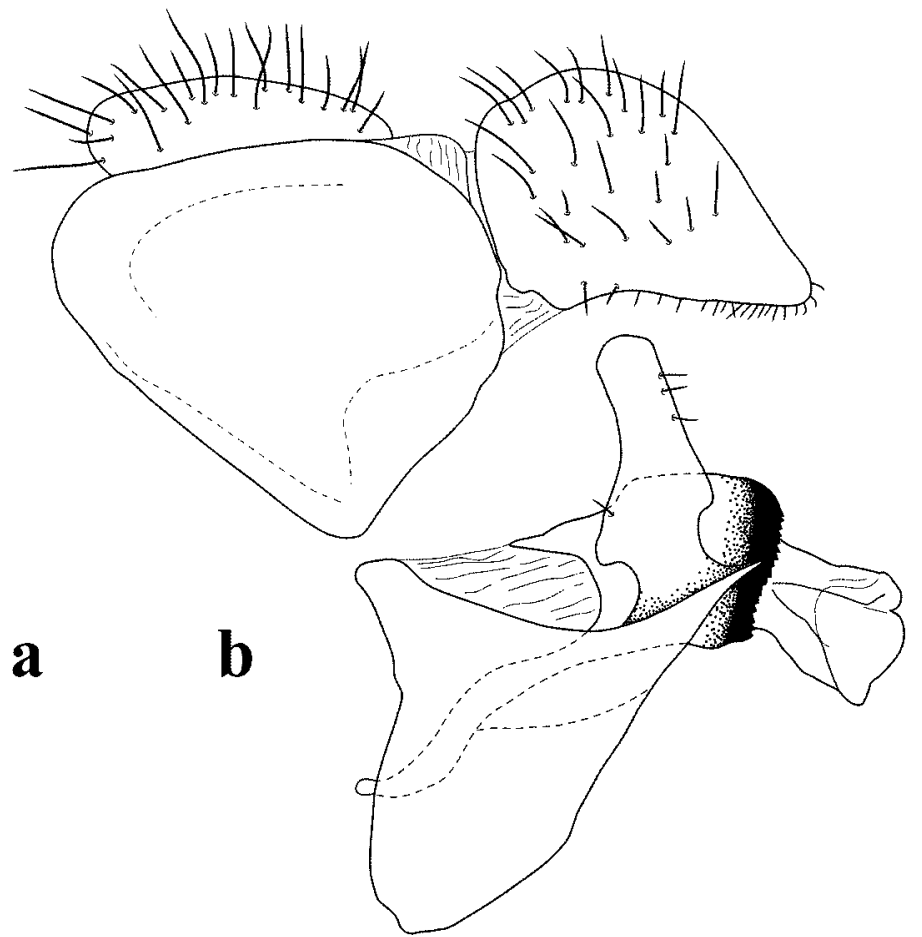

FIGURE 21. Male genitalia of Allograpta (Costarica) nishida sp. nov.: a, dorsal view; b, lateral view. 


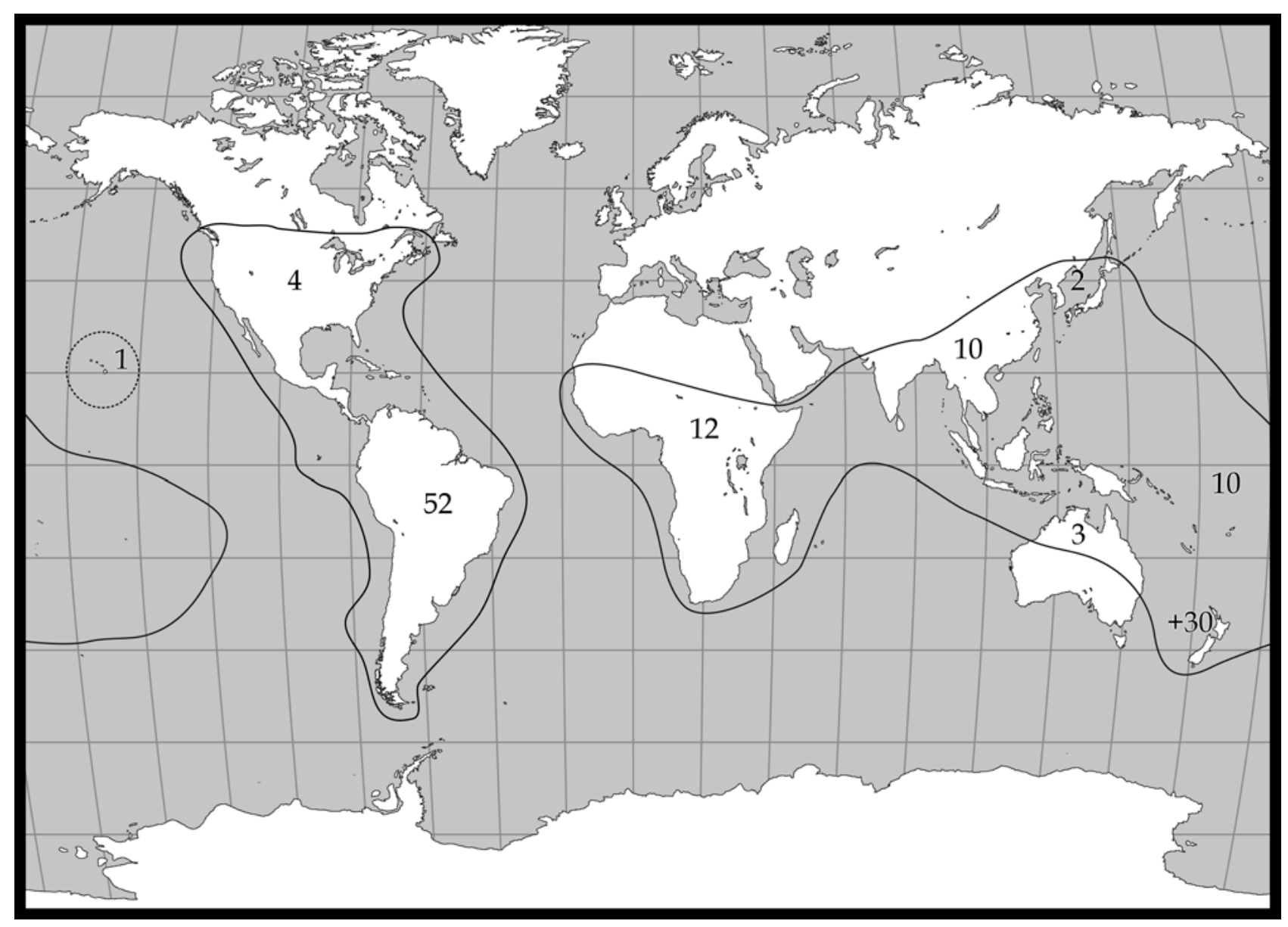

FIGURE 22. World distribution of the genus Allograpta, modified from Vockeroth (1969).

\section{Phylogenetic analysis}

A total of 22 ingroup taxa and 34 morphological characters were analyzed (see Tables 1 and 2). Parsimony was chosen as the optimality criterion for this study. The data matrix of morphological characters was analyzed in TNT (Goloboff et al. 2008) using the command ie (implicit enumeration) for an exact search. The analysis resulted in 5 equally parsimonious trees of 91 steps of length (C.I. $=0.42$, R.I. $=0.65)$ (figs. 25-29). The strict consensus tree is shown in figure 23. Figure 24 represents the agreement subtree, with the largest subset of taxa for which the relationships among them are invariant across all parsimonious trees. Figures were edited in Winclada (Nixon 2002). Bootstraping and Jackknife values were obtained with 1000 replications in TNT.

In the strict consensus tree, only 8 nodes were resolved with different support values. Two species of Sphaerophoria, S. (Loveridgeana) beattiei and S. (Sphaerophoria) novaeangliae, were resolved together in the strict consensus tree with low bootstrap and jackknife values, but more importantly S. scripta was never recovered with these 2 species of the same genus in any of the 5 equally parsimonious trees. Vockeroth (1969) indicated that the terminalia of S. novaeangliae and S. loewii do differ rather markedly from those of the other species. Previous molecular analyses (Mengual et al. 2008b) resolved S. loewii as the sister-taxon of Exallandra cinctifacies. Present results placed S. novaeangliae with the subgenus Loveridgeana, a fact that highlights the morphological differences between these two species of Sphaerophoria and the rest of the same subgenus. 


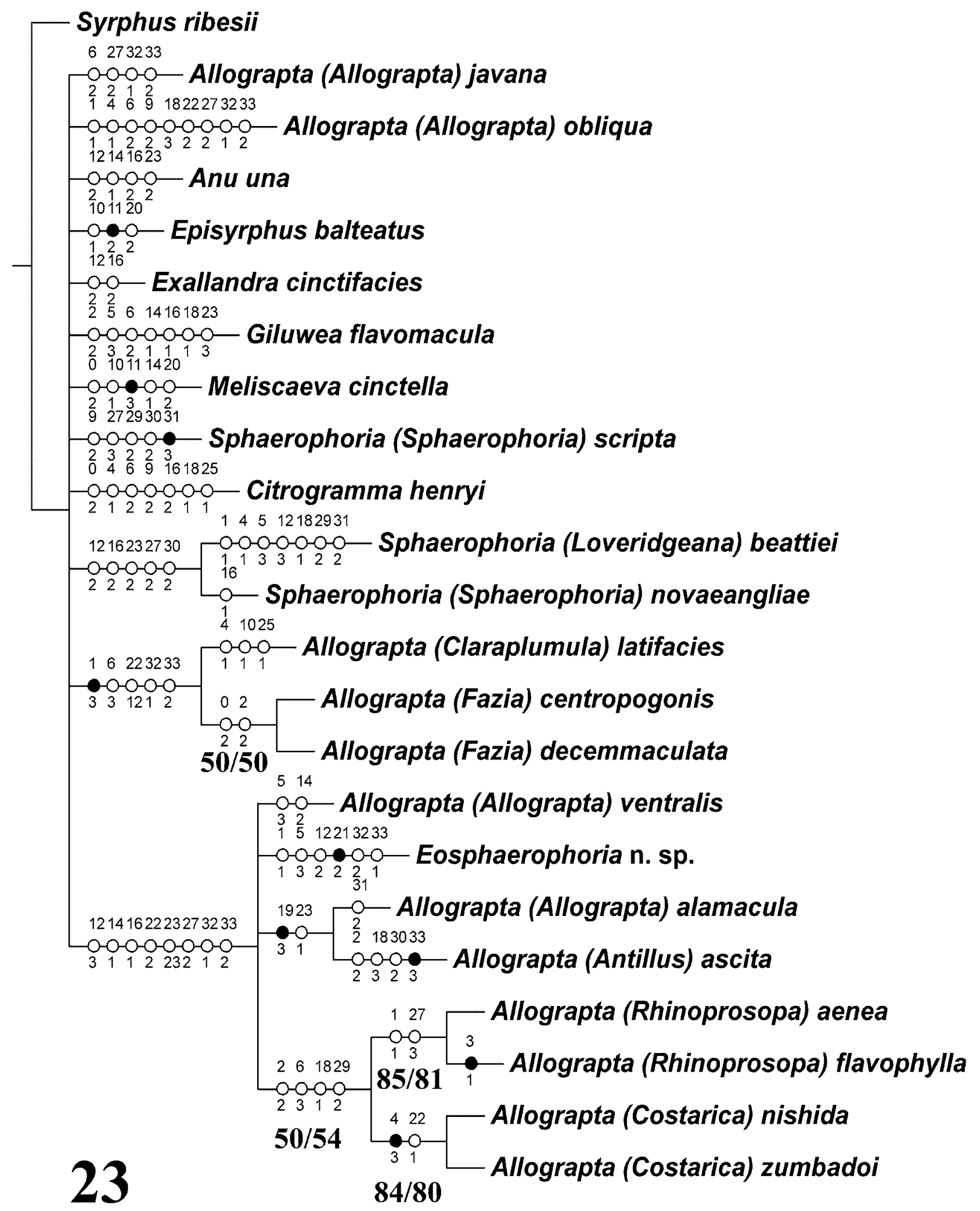

FIGURE 23. Strict consensus tree of five most parsimonious trees based on morphological characters using parsimony. Bootstrap and Jackknife support values above 50\% are indicated below branches (Boostrap/Jackknife). 

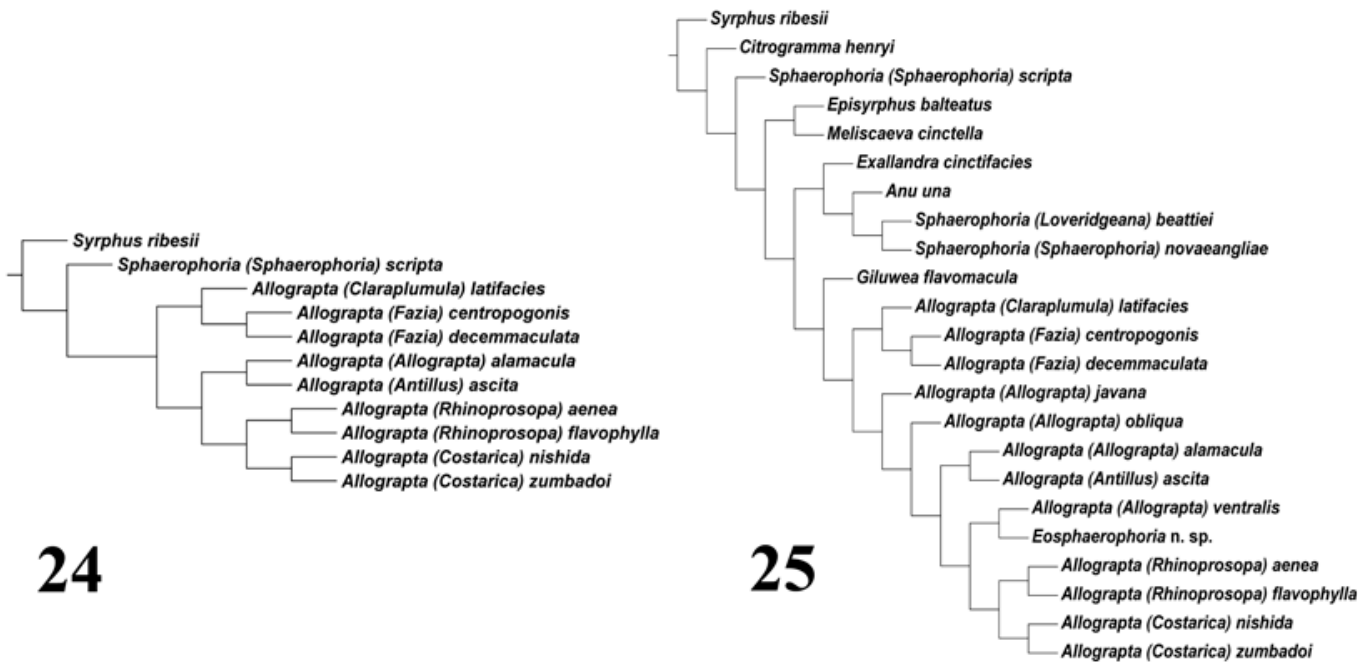

24
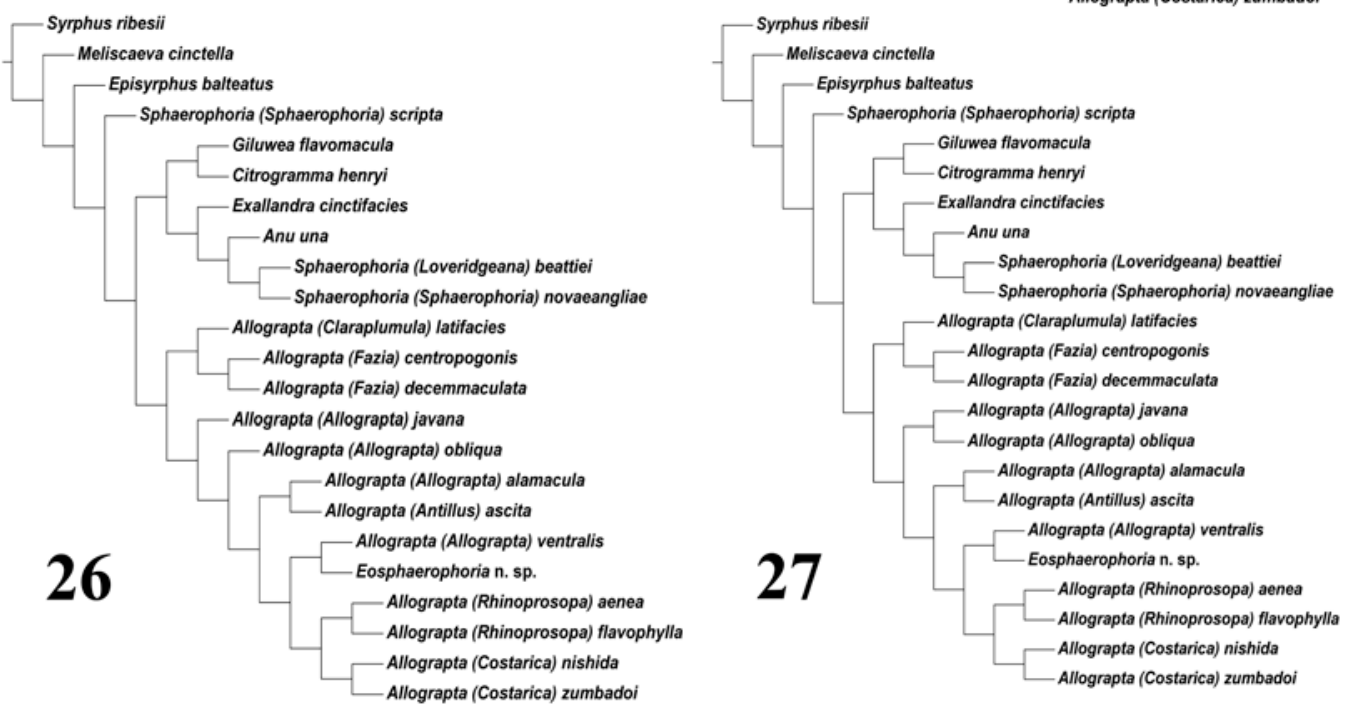

-Allograpta (Claraplumula) latifacies

- Allograpta (Fazia) centropogonis - Allograpta (Fazia) decemmaculata - Allograpta (Allograpta) javana

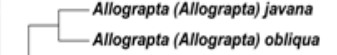

Allograpta (Allograpta) alamacula

27

Allograpta (Antillus) ascita

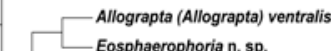

Eosphaerophoria n. sp.

Allograpta (Rhinoprosopa) aenea

Allograpta (Rhinoprosopa) flavophylla

- Allograpta (Costarica) nishida - Allograpta (Costarica) zumbadoi
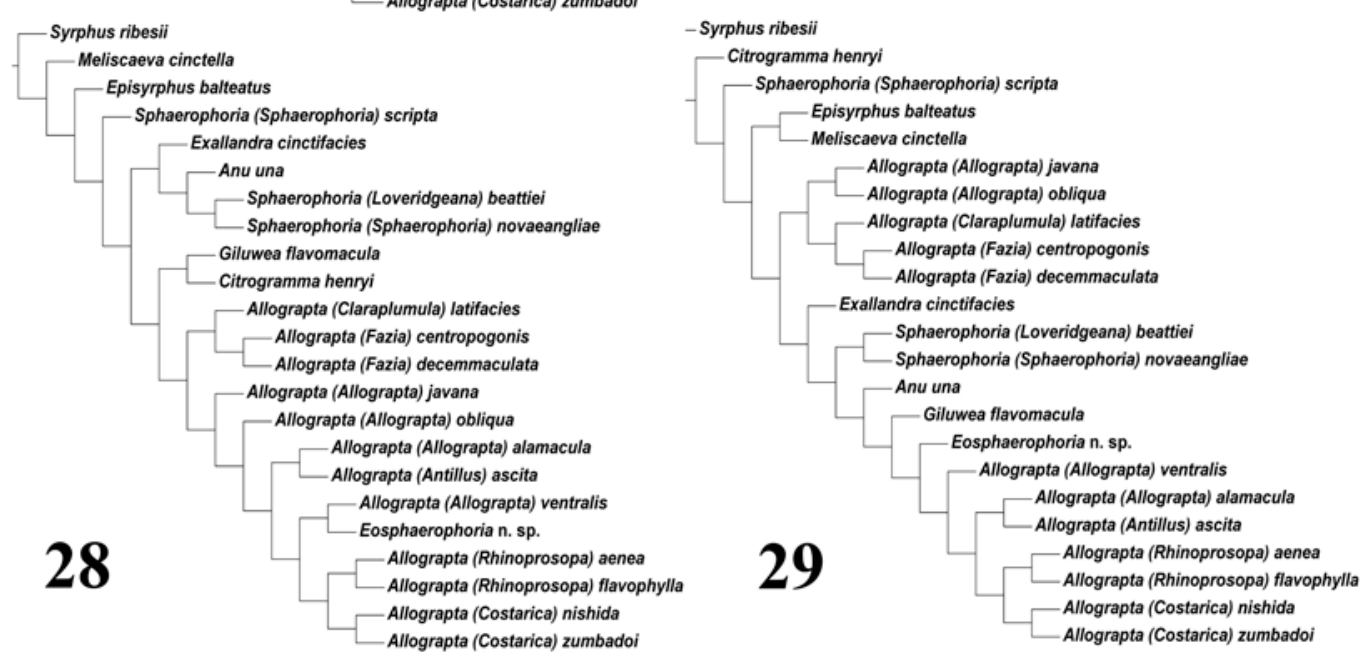

FIGURES 24-29. Cladograms of Allograpta relationships. 24. Agreement subtree; 25-29. The individual most parsimonious trees resulted from the analysis of morphological characters. 
The genus Allograpta was resolved into several species groups in the strict consensus tree. The species of Allograpta sensu stricto, A. obliqua and A. javana, were placed in the basal polytomy in the consensus tree, but looking at the 5 equally parsimonious trees, in the majority of the cases they form a clade with the rest of Allograpta species including Eosphaerophoria.

Two groups of Allograpta species are found in the strict consensus tree: one group with the species of the subgenus Fazia with the subgenus Claraplumula, and a second group with A. (Allograpta) ventralis, A. (Allograpta) alamacula, the representatives of the subgenera Costarica, Antillus and Rhinoprosopa, and the genus Eosphaerophoria. Subgenera Costarica and Rhinoprosopa were recovered in the strict consensus tree and related. The placement of A. alamacula with A. (Antillus) ascita probably is due to the presence of a dark macula in the wing. These results show that inclusion of more taxa and/or additional morphological characters are necessary to increase the topological resolution and clade support values. Although the monophyly of the genus Allograpta is questionable based on our results, some groups were consistently recovered (see fig. 24). The combination of morphological and molecular data is vital for a better supported hypothesis of the phylogenetic relationships among the included genera and to generate a stable classification.

\section{Acknowledgments}

This work is the first of many planned on the syrphine clade that includes Allograpta. Future papers will address the phylogeny of the group based on morphology and DNA characters (Mengual et al. in prep.) as well as a revision of the Neotropical species (Ruiz et al., in prep.). The order of authorship is merely chronological by age; the junior authors in age contributed the new insights and inspiration for the paper and the senior the history and "wisdom!"

The color habitus drawings [figs. 1, 4-6] were provided by Taina Litwak except that of A. (Costarica) zumbadoi [fig. 3], which was drawn by Jennifer Fairman and reproduced from Thompson et alia (2000) and $A$. (Fazia) centropogonis [fig. 2], which was drawn by Leonardo Donzo and reproduced from Nishida et alia (2002). The black and white line drawings were from Hull (1949b) [figs. 7-10], Thompson et al. (2000) [fig. 14], Carver \& Thompson (2003) [fig. 15] and Vockeroth (1969 [figs. 11-13], 1973 [fig. 16]), Taina Litwak (new) [figs. 17-19] and Ximo Mengual (new) [figs. 20-21]. The distribution map [fig. 22] was produced by Charyn Michel.

We thank Wayne N. Mathis, Department of Entomology, Smithsonian Institution, Washington, and Natalia Vanderberg, Allen Norrbom, and Alma Solis, Systematic Entomology Laboratory, USDA, Washington, for their critical reviews of the manuscript. The original version of the manuscript was produced and submitted while the senior author (in age, that is) was an employee of the US Department of Agriculture. Major revisions were made after his forced retirement and while he was an adjunct and emeritus scientist with the Smithsonian Institution, where Ximo Mengual is now currently a post-doctoral research fellow supported by the Schlinger Foundation. So, we acknowledge their continued support and acknowledge USDA decision to no longer support research on flower flies.

\section{References}

Aldrich, J.M. (1905) A catalogue of North American Diptera. Smithsonian Miscellaneous Collections, 46,680 pp. Washington. [before 1905.05.25]

Bankowska, R. (1962) Studies on the family Syrphidae (Diptera). Helenomyia gen. nov. Bulletin de l'Académie Polonaise des Sciences (Série des Sciences Biologiques), 10, 311-314. [1962.09.??]

Bezzi, M. (1915) The Syrphidae of the Ethiopian Region based on material in the collection of the British Museum (Natural History), with descriptions of new genera and species. British Museum (Natural History), London, 146 pp. [before 1915.08.11 date received at Smithsonian Institution]

Bezzi, M. (1920) Syrphidae aethiopicae Musei nationalis hungarici [part]. Broteria (Zoologica), 18, $131-142$. 
[1920.10.??]

Bezzi, M. (1923) Bombyliidae et Syrphidae. Résultats scientifiques. Voyage de Ch. Alluaud et R. Jeannel en Afrique orientale (1911-1912), [Diptera 6] 53, 315-351. [1923.04.15]

Bezzi, M. (1928) Diptera Brachycera and Athericera of the Fiji Islands based on material in the British Museum (Natural History). British Museum (Natural History), London, viii + 220 pp. [1928.06.23]

Bigot, J.M.F. (1857) Dípteros.. In: Sagra, R. de la (Eds), Historia fisica, politica y natural de la Isla de Cuba. Segunda parte. Historia natural. Vol. VII. Crustaceos, aragnidos é insectos. A. Bertrand, Paris, pp. 328-349, pl. 20. [after 1857.10.10]

Bigot, J.M.F. (1883) Diptères nouveaux ou peu connus. 21e partie. XXXII. Syrphidi (1re partie). Annales de la Société Entomologique de France, 3 (6), 221-258. [1883.10.31]

Bigot, J.M.F. (1884) Dipteres nouveaux ou peu connus. 24 partie, XXXII: Syrphidi (2 partie). Especes nouvelles, no. III. Annales de la Société Entomologique de France, (6) 4, 73-80, 81-116. [1884.10.08]

Bigot, J.M.F. (1889) Dipteres nouveaux ou peu connus. 34e partie, XLII: Diagnoses de nouvelles especes. Annales de la Société Entomologique de France, 8 (6), 253-270. [1889.01.09]

Blanchard, E. (1852) Orden IX. Dípteros. In: Gay, C. (Eds), Historia física y política de Chile. Zoologia. Vol. 7. Published by Author, Paris \& Museo de Historia Natural, Santiago, pp. 327-468. [before 1852.12.15]

Bowie, M.H. (2001) Ecology and morphology of Allograpta ventralis (Diptera: Syrphidae) a predator of the cabbage tree mealybug Balanococcus cordylinidis (Hemiptera: Pseudococcidae). New Zealand Natural Sciences, $26,1-11$. [before 2002.08.07 receipt SIL]

Brammer, C.A. \& Dohlen, C.D. von (2007) Evolutionary history of Stratiomyidae (Insecta: Diptera): The molecular phylogeny of a diverse family of flies. Molecular Phylogenetics and Evolution, 43, 660-673. [2007.??.??]

Brown, R.W. (1956) Composition of scientific words, a manual of methods and a lexicon of materials for the practice of logotechnics. Published by the author, Baltimore, 882 pp. [1956.??.??]

Brunetti, E. (1913) Zoological results of the Arbor Expedition, 1911-12. XI. Diptera. Records of the Indian Museum, 8, 149-190, pl. 6. [1913.??.??]

Bybee, M.S., Taylor, S.D., Nelson, C.R., Whiting, M.F. (2004) A phylogeny of robber flies (Diptera: Asilidae) at the subfamilial level: molecular evidence. Molecular Phylogenetics and Evolution, 30, 789-797. [2004.??.??]

Carver, M. \& Thompson, F.C. (2003) Two new species of Syrphidae (Diptera) from Australia. Studia Dipterologica, 10, 37-41. [2003.12.15]

Cheng, X., Lu, J., Huang, C., Zhou, H., Dai, Z. \& Zhang, G. (2000) Determination of phylogenetic position of Pipizini (Diptera: Syrphidae): based on molecular, biological and morphological data. Science in China (Series C), 42, 146156. [2000.??.??]

Curran, C.H. (1925a) New American Diptera. II. Annals and Magazine of Natural History, 16 (9), $338-354$. [1925.10.01]

Curran, C.H. (1925b) Contribution to a monograph of the American Syrphidae from north of Mexico. Kansas University Science Bulletin, 15, 7-216. [1925.12.01]. The title page for this article shows the date "December, 1924" and the title page for the whole volume shows the date "April 1, 1925." The copy in the Smithsonian Institution library is dated as received on 4 January 1926. However, I have seen a number of reprints dated in Curran's own hand as "Issued Dec. 1, 1925". This is the date we have accepted.

Curran, C.H. (1927a) New Neotropical and Oriental Diptera in the American Museum of Natural History. American Museum Novitates, 245, 9 pp. [1927.01.27]

Curran, C.H. (1927b) Diptera of the American Museum Congo Expedition. Part I. -- Bibionidae, Bombyliidae, Dolichopodidae, Syrphidae and Trypaneidae. Bulletin of the American Museum of Natural History, 57, 33-89. [1927.11.09]

Curran, C.H. (1928) The Syrphidae of the Malay Peninsula. Journal of the Federated Malay States Museum, 14, 141324. [1928.09.??]

Curran, C.H. (1931) Records and descriptions of Syrphidae from North Borneo including Mt. Kinabalu. Journal of the Federated Malay States Museum, 16, 333-376. [1931.07.??]

Curran, C.H. (1932) New American Syrphidae (Diptera), with notes. American Museum Novitates, 519,9 pp. [1932.03.31]

Curran, C.H. (1934) The families and genera of North American Diptera. Ballou Press, New York, 512 pp., 2 pls. [1934.08.25]

Curran, C.H. (1936) New Neotropical Syrphidae (Diptera). American Museum Novitates, 882, 17 pp. [1936.10.09]

Curran, C.H. (1938) Records and descriptions of African Syrphidae -- II (Diptera). American Museum Novitates, 1010 , 20 pp. [1938.12.30]

Doesburg, P.H. van (1958) Syrphiden-allerlei. Entomolgische Berichten, 18, 41-46. [1958.??.??]

Doesburg, P.H. van (1966) Syrphidae from Suriname. Additional records and descriptions. Studies on the Fauna of Suriname and other Guyanas (Uitg. Natuurwet. Studkring. Suriname), 9 (35), 61-107. [1966.??.??]

Doesburg, P.H. van (Sr.) \& Doesburg, P. H. van (Jr.) (1977) La faune terrestre de l'Ile de Sainte-Helene. Troisieme partie. 
13. Fam. Syrphidae. Annales du Musee Royal de l'Afrique Centrale, Série in 8o, Sciences zoologiques, 215 (1976): 63-74. [1977.??.??]

Dušek, J, \& Láska, P. (1967) Versuch zum Aufbau eines natürlichen Systems mitteleuropäischer Arten der Unterfamilie Syrphinae (Diptera). Acta Scientiarum Naturalium Academiae Scientiarum Bohemoslovacae, Brno (Nova Series), 1, 349-390. [1967.03.16]

Enderlein, G. (1922) Ein neues Tabanidensystem. Mitteilungen aus dem Zoologischen Museum in Berlin, 10, 333-351. [1922.08.??]

Enderlein, G. (1938a) Beitrag zur Kenntnis der Syrphiden. Sitzungsberichte der Gesellschaft Naturforschender Freunde zи Berlin, 1937, 192-237. [1938.03.01]

Enderlein, G. (1938b) Die Dipterenfauna der Juan-Fernandez-Inseln und der Oster-Insel. In: Skottsberg, K. (Eds), The Natural History of Juan Fernandez and Easter Island. Volume III. Zoology, pp. 643-680. [1938.10.13]

Evenhuis, N.L., Pape, T., Pont, A.C. \& Thompson, F.C. (Eds) (2008) Biosystematic Database of World Diptera. Available from http://www.diptera.org/names (15 May 2008).

Fabricius, J.C. (1805) Systema antliatorum secundum ordines, genera, species... C. Reichard, Brunsvigae [=Brunswick], xiv $+15-372+[1]+30$ pp. [1805.??.??]

Fluke, C.F., Jr. (1956-7) Catalogue of the family Syrphidae in the Neotropical Region. Revista Brasileira de Entomologia, 6, 193-268 [1956.12.10]; 7: 1-181. [1957.06.20]

Fluke, C.L., Jr. (1931) Notes on certain Syrphus flies related to Xanthogramma (Diptera Syrphidae) with descriptions of two new species. Transaction of the Wisconsin Academy of Sciences, Arts and Letters, 26, 289-309, 2 pls. [before 1931.07.29 receipt SIL]

Fluke, C.L., Jr. (1933) Revision of the Syrphus flies of America north of Mexico (Diptera, Syrphidae, Syrphus s. l.). Part I. Transactions of the Wisconsin Academy of Sciences, Arts and Letters, 28, 63-127, 3 pls. [before 1933.06.28 receipt SIL]

Fluke, C.L., Jr. (1935) Revision of the Epistrophe flies of America north of Mexico (Diptera, Syrphidae). Entomologica Americana, 15, 1-56, 3 pls. [1935.??.??]

Fluke, C.L., Jr. (1942) Revision of the Neotropical Syrphini related to Syrphus (Diptera, Syrphidae). American Museum Novitates, 1201, 24 pp. [1942.10.19]

Fluke, C.L., Jr. (1950) The male genitalia of Syrphus, Epistrophe and related genera (Diptera, Syrphidae). Transactions of the Wisconsin Academy of Sciences, Arts and Letters, 40, 115-148. [1950.08.15]

Fluke, C.L., Jr. (1951) Corrections to the paper on "The male genitalia of Syrphus, Epistrophe and related genera" (Diptera: Syrphidae). Entomological News, 62, 217-218. [1951.??.??]

Frey, R. (1946) Ubersicht der Gattungen der Syrphiden-Unterfamilie Syrphinae (Syrphine + Bacchinae). Notulae Entomologicae, 25, 152-172. [1946.04.01]

Ghorpade, K. (1994) Diagnostic keys to new and known genera and species of Indian subcontinent Syrphini (Diptera: Syrphidae). Colemania. Insect Biosystematics, 3, 15 pp. [1994.12.??]

Giglio-Tos, E. (1892) Diagnosi di nuove specie di Ditteri. VII. Sirfidi del Messico. Bollettino del Museo di Zoologia ed Anatomia Comparata della R. Università di Torino, 7 (132), 5 pp. [1892.10.20]

Giglio-Tos, E. (1893) Ditteri del Messico. Parte II. Syrphidae - Conopidae - Pipunculidae. C. Clausen, Torino, 80 pp., 1 pl. [1893.03.31]

Goloboff, P., Farris, J.S. \& Nixon, K.C. (2008) T.N.T.: Tree Analysis Using New Technology. Program and documentation, Tucumán, Argentina.

Harris, T.W. (1835) Insects. In: Hitchcock, E. (Eds), Report on the geology, mineralogy, botany, and zoology of Massachusetts. 2nd Edition. J. S. \& C. Adams, Amherst, pp. 553-602. [1835.??.??]

Herve-Bazin, J. (1923) Diagnoses de Syrphides [Dipt.] nouveaux du Laos (Indo-Chine francaise). Bulletin de la Société entomologique de France, 1923, 25-28. [1923.??.??]

Hippa, H. \& Ståhls, G. (2005) Morphological characters of adult Syrphidae: descriptions and phylogenetic utility. Acta Zoologica Fennica, 215, 1-72. [2005.11.24]

Hull, F.M. (1937a) New species of exotic syrphid flies. Psyche, 44, 12-32. [1937.??.??]

Hull, F.M. (1937b) Some Neotropical and Oriental Syrphid flies in the United States National Museum. Journal of the Washington Academy of Sciences, 27, 165-176. [1937.04.15]

Hull, F.M. (1941) Some flies of the family Syrphidae. Mesogramma. Psyche, 48, 45-49. [1941.??.??]

Hull, F.M. (1942) Some flies of the genus Mesogramma. Proceedings of the New England Zoological Club, $20,17-24$. [1942.??.??]

Hull, F.M. (1943a) Some new American syrphid flies (Diptera). Entomological News, 54, 29-37. [1943.03.12]

Hull, F.M. (1943b) New species of Baccha and related flies. Entomological News, 54, 135-140. [1943.??.??]

Hull, F.M. (1943c) New species of the genera Baccha and Rhinoprosopa (Syrphidae). Journal of the Washington Academy of Sciences, 33, 214-216. [before 1943.07.13 receipt SIL]

Hull, F.M. (1944a) Some flies of the family Syrphidae in the British Museum (Natural History). Annals and Magazine of natural History, 11 (11), 21-61. [1944.01.??] 
Hull, F.M. (1944b) Studies on syrphid flies in the Museum of Comparative Zoology. Psyche, 51, 22-45. [1944.??.??]

Hull, F.M. (1947) Some American syrphid flies. Psyche, 54, 230-240. [1947.??.??]

Hull, F.M. (1949a) The genus Baccha from the New World. Entomologica Americana, 27, 89-291. [1949.04.30]

Hull, F.M. (1949b) The morphology and inter-relationship of the genera of syrphid flies, recent and fossil. Transaction of the Zoological Society of London, 26, 257-408. [1949.05.??]

Hull, F.M. (1949c) American syrphid flies of the subfamilies Cheilosinae and Syrphidae. Bulletin of the Brooklyn Entomological Society, 44, 73-79. [1949.06.08]

Hull, F.M. (1949d) Studies upon Diptera in the British Museum. Annals and Magazine of Natural History, 2 (12), $727-$ 746. [1949.11.04]

International Commission on Zoological Nomenclature (1999) International Code of Zoological Nomenclature. 4th Edition. The International Trust for Zoological Nomenclature, London, 306 pp. [1999.??.??]

Kassebeer, C.F. (2000) Die Schwebfliegen (Diptera, Syrphidae) von La Reunion, mit Anmerkungen zur Fauna der Madagassis. Dipteron 3, 43-66. [2000.11.24]

Keiser, F. (1971) Syrphidae von Madagaskar (Dipt.). Verhandlungen der Naturforschenden Gesellschaft in Basal, 81, 223-318. [1971.11.??]

Kertész, K. (1899) Verzeichniss einiger, von L. Biro in Neu-Guinea und am malayischen Archipel gesammelten Dipteren. Természetrajzi Füzetek, 22, 173-195. [1899.03.10]

Kertész, K. (1910) Catalogus dipterorum hucusque descriptorum. Vol. 7. Museum Nationale Hungaricum, Budapestini [=Budapest], 470 pp. [1910.06.??]

Knutson, L.V., Thompson, F.C. \& Vockeroth, J.R. (1975) Family Syrphidae. In: Delfinado, M. D. \& Hardy, D. E. (Eds), A catalog of the Diptera of the Oriental Region. Vol. II. Suborder Brachycera through division Aschiza, suborder Cyclorrhapha. University of Hawaii Press, Honolulu, pp. 307-374. [1975.10.05]

Kutty, S.N., Bernasconi, M.V., Šifner, F. \& Meier, R. (2007) Sensitivity analysis, molecular systematics and natural history of Scathophagidae (Diptera: Cyclorrhapha: Calyptratae). Cladistics, 23, 64-83. [before 2007.05.02 receipt SIL]

Linnaeus, C. (1758) Systema naturae per regna tria naturae, secundum classes, ordines, genera, species, cum caracteribus, differentiis, synonymis, locis. Ed. 10, Vol. 1. L. Salvii, Holmiae [= Stockholm], 824 pp. [1758.01.01]

Loew, H. (1858) Bidrag till kannedomen om Afrikas Diptera [part]. Öfversigt af Kongliga Vetenskaps-Akademiens Förhandlingar, Stockholm, 14, 337-383. [early 1858.??.??]

Lynch Arribálzaga, F. (1892) Dipterologia Argentina, Syrphidae. [Part]. Anales de la Sociedad cientifica Argentina, 33, 51-58. [1892.??.??]

Macquart, J. (1842) Dipteres exotiques nouveaux ou peu connus. Tome deuxieme.--2e partie. Mémoires de la Société (Royale) des Sciences, de l' Agriculture et des Arts, Lille, 1841 (1), 65-200, 22 pls. [1842.??.??]

Macquart, J. (1846) Dipteres exotiques nouveaux ou peu connus. Supplement. [1]. Mémoires de la Société (Royale) des Sciences, de l' Agriculture et des Arts, Lille, 1844, 133-364, 20 pls. [before 1846.07.22]

Macquart, J. (1850) Dipteres exotiques nouveaux ou peu connus. 4e supplement. Mémoires de la Société (Royale) des Sciences, de l' Agriculture et des Arts, Lille, 1849, 309-479, pls. 1-14. [before 1850.07.06]

Masetti, A., Luchetti, A., Sommaggio, D., Burgio, G. \& Mantovani, B. (2006) Phylogeny of Chrysotoxum species (Diptera: Syrphidae) inferred from morphological and molecular characters. European Journal of Entomology, 103, 459-467. [before 2006.05.18 receipt SIL]

Matsuda, R. (1970) Morphology and evolution of the insect thorax. Memoirs of the Entomological Society of Canada, 76, 431 pp. [1970.??.??]

Matsumura, S. (1918) New species of the economic Syrphidae of Japan. Journal of the College of Agriculture, Hokkaido imperial University, 8, 1-31, pl. 1. [1918.10.??]

Meigen, J.W. (1822) Systematische Beschreibung der bekannten europaischen zweiflugeligen Insekten. Dritter Theil. Schulz-Wundermann, Hamm, x + 416 pp., pls. 22-32. [1822.09.??]

Meijere, J.C.H. de (1914) Studien uber sudostasiatische Dipteren. IX. Tijdschrift voor Entomologie, 57, 137-168 [1914.06.30], 169-175, pls. 5-7. [1914.10.15]

Meijere, J.C.H. de (1929) Fauna Buruana. Syrphiden nebst einigen Brachyceren Orthorrhaphen. Treubia, 7, $378-387$. [1929.04.??]

Mengual, X., Ståhls, G. \& Rojo, S. (2008a) First phylogeny of predatory flower flies (Diptera, Syrphidae, Syrphinae) using mitochondrial COI and nuclear 28S rRNA genes: Conflict and congruence with the current tribal classification. Cladistics, 24, 543-562. [2008.??.??]

Mengual, X., Ståhls, G. \& Rojo, S. (2008b) Molecular phylogeny of Allograpta (Diptera, Syrphidae) reveals Diversity of lineales and non-monophyly of phytophagous taxa. Molecular Phylogenetics and Evolution, 49, 715-727. [2008.??.??]

Milankov, V., Ståhls, G., Stamenković, J. \& Vujić, A. (2007) Genetic diversity of populations of Merodon aureus and M. cinereus species complexes (Diptera, Syrphidae): integrative taxonomy and implications for conservation priorities on the Balkan Peninsula. Conservation Genetics, 9 (5), 1125-1137. [2007.??.??] 
Milankov, V., Stamenković, J., Ludoški, J., Ståhls, G. \& Vujić, A. (2005) Diagnostic molecular markers and the genetic relationships among three species of the Cheilosia canicularis group (Diptera: Syrphidae). European Journal of Entomology, 102, 125-131. [before 2005.06.15 receipt SIL]

Miller, D.W. (1921) Material for a monograph on the Diptera fauna of New Zealand. part II, family Syrphidae. Transactions of the New Zealand Institute, 53, 289-333. [1921.08.08]

Miller, D.W. (1924) Material for a monograph on the Diptera fauna of New Zealand: part 2, family Syrphidae, supplement A. Transactions of the New Zealand Institue, 55, 281-284. [1924.06.18]

Mutin, V. (1986) New and little known species of hover flies (Diptera, Syrphidae) in the USSR fauna. Entomologicheskoe Obozrenie, 65, 826-832. [In Russian] [1986.??.??]

Nishida, K., Rotheray, G. \& Thompson, F.C. (2002) First non-predaceous syrphine flower fly (Diptera: Syrphidae): A new leaf-mining Allograpta from Costa Rica. Studia Dipterologia, 9, 421-436. [2003.04.15]

Nixon, K.C. (2002) Winclada. Version 10.00.08. Published by the author, Ithaca, New York.

Osten Sacken, C.R. (1875) A list of the North American Syrphidae. Bulletin of the Buffalo Society of Natural Science, 3 , 38-71. [1875.11.??]

Osten Sacken, C.R. (1877) Western Diptera: Descriptions of new genera and species of Diptera from the region west of the Mississippi and especially from California. Bulletin of the United States Geological and Geographic Survey of the Territories, 3, 189-354. [1877.04.30]

Osten Sacken, C.R. (1878) Catalogue of the described Diptera of North America. Smithsonian Miscellaneous Collection, 270, xlvii + 276 pp. [1878.10.??]

Peck, L.V. (1988) Family Syrphidae. In: Soos, A. (Es.), Catalogue of Palaearctic Diptera. Vol. 8. Akademiai Kiado, Budapest, pp. 11-230. [1988.09.20]

Philippi, R.A. (1865) Aufzahlung der chilenischen Dipteren. Abhandlungen der [K.-k. (=Kaiserlich-königlichen)] Zoologich-Botanischen Gesellschaft in Wien, 15, 595-782, 7 pls. [1865.??.??]

Rojo, S., Gilbert, F., Marcos-García, Ma A., Nieto, J.M. \& Mier, M.P. (2003) A world review of predatory hoverflies (Diptera, Syrphidae: Syrphinae) and their prey. Centro Iberoamericano de la Biodiversidad (CIBIO), Alicante, 319 pp. [about 2003.06.01]

Rondani, C. (1863) Diptera exotica revisa et annotata. Novis non nullis descriptis. E. Soliani, Modena, 99 pp., 1 pl. [1863.??.??]

Rotheray, G.E. \& Gilbert, F. (1989) The phylogeny and systematics of European predacious Syrphidae (Diptera) based on larval and puparial stages. Zoological Journal of the Linnean Society, 95, 29-70. [before 1989.02.24 receipt SIL]

Rotheray, G.E., Gilbert, F. (1999) Phylogeny of Palaearctic Syrphidae (Diptera): evidence from larval stages. Zoological Journal of the Linnean Society, 127, 1-112. [before 1999.10.07 receipt SIL]

Sack, P. (1941) Syrphidae (Dipt.). In: Titschack, E. (Eds), Beitrage zur Fauna Perus. Band 1. P. 97-120. [1941.??.??]

Say, T. (1823) Descriptions of dipterous insects of the United States. Journal of the Academy of Natural Sciences, Philadelphia, 3, 9-32 [1823.04.??], 33-64 [1823.05.??], 73-104 [1823.??.??].

Schiner, I.R. (1868) Diptera. In: Wullerstorf-Urbair, B. von (in charge), Reise der osterreichischen Fregatte Novara. Zoology 2 (1). B. K. Gerold's Sohn, Wien, vi + 388 pp., 4 pls. [1868.04.30]

Schuh, R.T., Weirauch, C. \& Wheeler, W.C. (2009) Phylogenetic analysis of family-group relationships in the Cimicomorpha (Hemiptera). Systematic Entomology, 34, 15-48. [2009.??.??]

Shannon, R.C. (1922) A reclassification of the subfamilies and genera of the North American Syrphidae. Bulletin of the Brooklyn Entomological Society, 16, 120-128 [1922.02.28]; 17, 30-43, pl. 2. [1922.06.15]

Shannon, R.C. (1923) A reclassification of the subfamilies and genera of North American Syrphidae (Diptera). Appendix. Bulletin of the Brooklyn Entomological Society, 18, 17-21. [1923.02.24]

Shannon, R.C. (1927) A review of the South American two-winged flies of the family Syrphidae. Proceedings of the United States National Museum, 70(9)[= No. 2658], 34 pp. [1927.04.29]

Shiraki, T. (1963) Diptera: Syrphidae. Insects of Micronesia, 13, 129-187. [1963.06.14]

Smith, K.G.V. \& Vockeroth, J.R. (1980) Family Syrphidae. In: Crosskey, R. W. (Eds), Catalogue of the Diptera of the Afrotropical Region. British Museum (Natural History), London, pp. 488-510. [1980.07.10]

Ståhls, G., Hippa, H., Rotheray, G., Muona, J. \& Gilbert, F. (2003) Phylogeny of Syrphidae (Diptera) inferred from combined analysis of molecular and morphological characters. Systematic Entomology, 28, 433-450. [before 2003.12.10 receipt SIL]

Ståhls, G., Vujic, A., Stuke, J.H., Doczkal, D. \& Muona, J. (2004) Phylogeny of the genus Cheilosia and the tribe Rhingiini (Diptera, Syrphidae) based on molecular and morphological characters. Cladistics, 4, 1-17. [2004.??.??]

Tao, C.C. \& Chiu, S.C. (1971) Biological control of citrus, vegetables and tobacco aphids. Special Publication, Taiwan Agricultural Research Institute, Taipei, 10, 110 pp. [1971.07.??]

Thompson, F.C. \& Vockeroth, J.R. (1989) Family Syrphidae. In: Evenhuis, N. L. (Eds), Catalog of the Diptera of the Australasian and Oceanian Regions. Bishop Museum Special Publication 86, Hawaii, pp. 437-458.. [1989.08.23]

Thompson, F.C. (1981) The flower flies of the West Indies (Diptera: Syrphidae). Memoirs of the Entomological Society of Washington, 9, 200 pp. [1981.09.02] 
Thompson, F.C. (1999a) Data Dictionary and Standards. Myia, 9, 49-63. [1999.01.??]

Thompson, F.C. (1999b) A key to the genera of the flower flies (Diptera: Syrphidae) of the Neotropical Region including descriptions of new genera and species and a glossary of taxonomic terms. Contributions on Entomology, International, 3, 321-378. [1999.08.23]

Thompson, F.C. (2008) A conspectus of New Zealand flower flies (Diptera: Syrphidae) with the description of a new genus and species. Zootaxa, 1716, 1-20. [2008.02.29]

Thompson, F.C., Thompson, B.J. \& Fairman, J.E. (2000) Only in Costa Rica: New Neotropical flower flies (Diptera: Syrphidae). Studia Dipterologica, 7, 33-43. [2000.09.15]

Thompson, F.C., Vockeroth, J.R. \& Sedman, Yale S. (1976) Family Syrphidae. Catalogue of the Diptera of America south of the United States, 46, 195 pp. [1976.08.09]

Thomson, C.G. (1869) Diptera. Species nova descripsit. In: Kongliga svenska fregatten Eugenies resa omkring jorden under befal af C. A. Virgin, åren 1851-1853. 2 (Zoologi) 1, Insecta. P. A. Norstedt \& Soner,Stockholm, pp. $443-614$. [after 1869.02.10, before 1869.03.10]

Vockeroth, J.R. (1969) A revision of the genera of the Syrphini (Diptera: Syrphidae). Memoirs of the Entomological Society of Canada, 62, 176 pp. [1969.07.15]

Vockeroth, J.R. (1973) Three additional synonyms of Allograpta (Diptera: Syrphidae). Canadian Entomologist, 105, 1101-1104. [1973.11.20]

Vockeroth, J.R. (1992) The flower flies of the subfamily Syrphinae of Canada, Alaska, and Greenland (Diptera: Syrphidae). Insects and Arachnids of Canada, 18. Research Branch, Agriculture Canada, Publication 1867, 456 pp. [1992.??.??]

Vujić, A., Ståhls, G., Rojo, S., Radenkovic, S. \& Simic, S. (2008) Systematics and phylogeny of the tribe Paragini (Diptera, Syrphidae) based on molecular and morphological characters. Zoological Journal of the Linnean Society 152(3): 507-536. [2008.??.??]

Walker, F. (1849) List of the specimens of dipterous insects in the collection of the British Museum. British Museum (Natural History), London, Part II, [iii]+231-484[1849.04.21]; Part III, [iii]+485-687 [1849.06.30]; Part IV, pp. [3] $+689-1172+[2] \cdot[1849.12 .08]$

Walker, F. (1852) Diptera. Part III, In: Saunders, W. W. (Eds), Insecta Saundersiana: or characters of undescribed insects in the collection of William Wilson Sauders, Esq., F.R.S., F.L.S., \& C. Vol. 1. Van Voorst, London, pp. 157-252, pls. 5-6. [before 1852.08.02]

Weng, J.L. \& Rotheray, G.E. (2009) Another non-predaceous syrphine flower fly (Diptera: Syrphidae): pollen feeding in the larva of Allograpta micrura. Studia Dipterologica, 14, in press.

Wiedemann, C.R.W. (1824) ... Analecta entomologica ... Regio Typographeo scholarum, Kiliae [=Kiel]. 60 pp., 1 pl. [1824.??.??]

Wiedemann, C.R.W. (1830) Aussereuropaische zweiflugelige Insekten. Zweiter Theil. Schulz, Hamm. xii + 684 pp., 5 pls. [1830.09.22]

Williston, S.W. (1882) Contribution to a monograph of the North American Syrphidae. Proceedings of the American Philosophical Society, 20, 299-332. [1882.08.03]

Williston, S.W. (1887) Synopsis of the North American Syrphidae. Bulletin of the United States National Museum, 31, $\mathrm{xxx}+335,12$ pls. [before 1887.06.30]

Williston, S.W. (1888) Synopsis of the families and genera of North American Diptera. Exclusive of the genera of the Nematocera and Muscidae, with bibliography and new species, 1878-88. J. T. Hathaway, New Haven, 84 pp. [1888.01.??]

Williston, S. W. (1896) Manual of North American Diptera. 2nd Ed. J. T. Hathaway, New Haven, liv + 167 pp. [1908.08.??]

Williston, S.W. (1908) Manual of North American Diptera. 3rd Ed. J. T. Hathaway, New Haven, 495 pp. [1908.08.29]

Wulp, F.M. van der (1867) Eenige Noord-Americaansche Diptera. Tijdschrift voor Entomologie, 10, 125-164, pl. 3-5. [1867.07.13]

Zuijen, M.P. van \& Nishida, K. (2009) Description of life history and immature stages of phytophagous flower fly, Allograpta zumbadoi Thompson (Diptera: Syrphidae: Syrphinae). Studia Dipterologica, 14, in press. 